\title{
Compositional Verification and Optimization of Interactive Markov Chains
}

\author{
Holger Hermanns ${ }^{1}$, Jan Krčál ${ }^{2}$, and Jan Křetínskýg ${ }^{2,3}$ \\ 1 Saarland University - Computer Science, Saarbrücken, Germany \\ hermanns@cs.uni-saarland.de \\ 2 Faculty of Informatics, Masaryk University, Czech Republic \\ $\{$ krcal,jan.kretinsky\}@fi.muni.cz \\ ${ }^{3}$ Institut für Informatik, Technical University Munich, Germany
}

\begin{abstract}
Interactive Markov chains (IMC) are compositional behavioural models extending labelled transition systems and continuous-time Markov chains. We provide a framework and algorithms for compositional verification and optimization of IMC with respect to time-bounded properties. Firstly, we give a specification formalism for IMC. Secondly, given a time-bounded property, an IMC component and the assumption that its unknown environment satisfies a given specification, we synthesize a scheduler for the component optimizing the probability that the property is satisfied in any such environment.
\end{abstract}

\section{Introduction}

The ever increasing complexity and size of systems together with software reuse strategies naturally enforce the need for component based system development. For the same reasons, checking reliability and optimizing performance of such systems needs to be done in a compositional way. The task is to get useful guarantees on the behaviour of a component of a larger system. The key idea is to incorporate assumptions on the rest of the system into the verification process. This assume-guarantee reasoning is arguably a successful divide-andconquer technique in many contexts MC81|AH96 HMP01.

In this work, we consider a continuous-time stochastic model called interactive Markov chains (IMC). First, we give a language for expressing assumptions about IMC. Second, given an IMC, an assumption on its environment and a property of interest, we synthesize a controller of the IMC that optimizes the guarantee, and we compute this optimal guarantee, too.

Interactive Markov chains are behavioural models of probabilistic systems running in continuous real time appropriate for the component-based approach HK09. IMC have a well-understood compositional theory rooted in process algebra, and are in use as semantic backbones for dynamic fault trees, architectural description languages, generalized stochastic Petri nets and Statemate extensions, see [HK09] for a survey. IMC are applied in a large spectrum of practical applications, ranging from water treatment facilities $\left[\mathrm{HKR}^{+} 10\right.$ to ultra-modern satellite designs $\mathrm{EKN}^{+} 12$. 
IMC arise from classical labelled transition systems by incorporating the possibility to change state according to a random delay governed by a negative exponential distribution with a given rate, see transitions labelled 1, 2 and 3 in the figure. Apart from delay expirations, state transi-

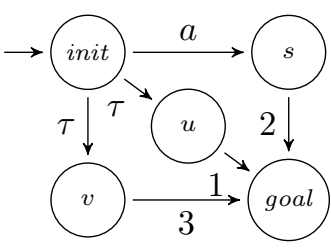
tions may be triggered by the execution of internal $(\tau)$ actions or external (synchronization) actions. Internal actions are assumed to happen instantaneously and therefore take precedence over delay transitions. External actions are the process algebraic means for interaction with other components, see $a$ in the figure. By dropping the delay transitions, labelled transition systems are regained in their entirety. Dropping action-labelled transitions instead yields continuoustime Markov chains - one of the most used performance and reliability models.

The fundamental problem in the analysis of IMC is that of time-bounded reachability. It is the problem to approximate the probability that a given set of states is reached within a given deadline. We illustrate the compositional setting of this problem in the following examples.

Examples. In the first example, consider the IMC $\mathcal{C}$ from above and an unknown environment $\mathcal{E}$ with no assumptions. Either $\mathcal{E}$ is initially not ready to synchronize on the external action $a$ and thus one of the internal actions is taken, or $\mathcal{E}$ is willing to synchronize on $a$ at the beginning. In the latter case, whether $\tau$ or $a$ happens is resolved non-deterministically. Since this is out of control of $\mathcal{C}$, we must assume the worst case and let the environment decide which of the two options will happen. For more details on this design choice, see $\mathrm{BHK}^{+} 12$. If there is synchronization on $a$, the probability to reach goal within time $t=1.5$ is $1-e^{-2 t} \approx 0.95$. Otherwise, $\mathcal{C}$ is given the choice to move to $u$ or $v$. Naturally, $v$ is the choice maximizing the chance to get to goal on time as it has a higher rate associated. In this case the probability amounts to $1-e^{-3 t} \approx 0.99$, while if $u$ were chosen, it would be only 0.78 . Altogether, the guaranteed probability is $95 \%$ and the strategy of $\mathcal{C}$ is to choose $v$ in init.

The example depicted on the right illustrates the necessity of assumptions on the environment: As it is, the environment can drive the component

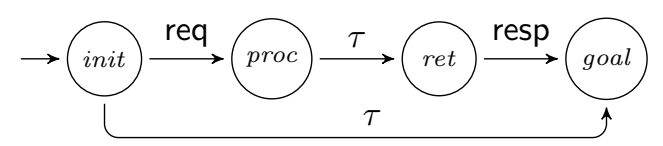
to state ret and let it get stuck there by not synchronising on resp ever. Hence no better guarantee than 0 can be derived. However, this changes if we know some specifics about the behaviour of the environment: Let us assume that we know that once synchronization on req occurs, the environment must be ready to synchronise on resp within some random time according to, say, an exponential distribution with rate 2 . Under this assumption, we are able to derive a guarantee of $95 \%$, just as in the previous example.

Observe the form of the time constraint we imposed in the last example: "within a random time distributed according to $\operatorname{Exp}(2)$ " or symbolically $\nabla_{\leq \operatorname{Exp}(2)} \varphi$. We call this a continuous time constraint. If a part of the environment is e.g. a model of a communication network, it is clear we cannot impose hard bounds (discrete time constraints) such as "within 1.5" as in e.g. a formula of MTL 
$\nabla_{\leq 1.5} \varphi$. Folklore tells us that messages might get delayed for longer than that. Yet we want to express high assurance that they arrive on time. In this case one might use e.g. a formula of CSL $\operatorname{Pr}_{\geq 0.95}\left(\nabla_{\leq 1.5} \varphi\right)$. However, consider now a system with two transitions labelled with resp in a row. Then this CSL formula yields only a zero guarantee. By splitting the time 1.5 in halves, the respective $\operatorname{Pr}_{\geq 0.77}\left(\diamond_{\leq 0.75} \varphi\right)$ yields only the guarantee $0.77^{2}=0.60$. The actual guarantee 0.80 is given by the convolution of the two exponential distributions and as such can be exactly obtained from our continuous time constraint $\nabla_{\leq \operatorname{Exp}(2)} \varphi$.

Our contribution is the following:

1. We introduce a specification formalism to express assumptions on continuoustime stochastic systems. The novel feature of the formalism are the continuous time constraints, which are vital for getting guarantees with respect to time-bounded reachability in IMC.

2. We incorporate the assume-guarantee reasoning to the IMC framework. We show how to synthesize $\epsilon$-optimal schedulers for IMC in an unknown environment satisfying a given specification and approximate the respective guarantee.

In our recent work $\mathrm{BHK}^{+} 12$ we considered a very restricted setting of the second point. Firstly, we considered no assumptions on the environment as the environment of a component might be entirely unknown in many scenarios. Secondly, we were restricted to IMC that never enable internal and external transitions at the same state. This was also a severe limitation as this property is not preserved during the IMC composition process and restricts the expressivity significantly. Both examples above violate this assumption. In this paper, we lift the assumption.

Each of the two extensions shifts the solution methods from complete information stochastic games to (one-sided) partial observation stochastic games, where we need to solve the quantitative reachability problem. While this is undecidable in general, we reduce our problem to a game played on an acyclic graph and show how to solve our problem in exponential time. (Note that even the qualitative reachability in the acyclic case is PSPACE-hard CD10.)

Related work. The synthesis problem is often stated as a game where the first player controls a component and the second player simulates an environment RW89. Model checking of open systems, i.e. operating in an unknown environment, has been proposed in KV96. There is a body of work on assumeguarantee reasoning for parallel composition of real-time systems [TAKB96 HMP01]. Lately, games with stochastic continuous-time have gained attention, for a very general class see BF09. While the second player models possible schedulers of the environment, the structure of the environment is fixed there and the verification is thus not compositional. The same holds for $\operatorname{Spr} 11 \mathrm{HNP}^{+} 11$, where time is under the control of the components.

A compositional framework requires means for specification of systems. A specification can be also viewed as an abstraction of a set of systems. Three valued abstractions stemming from [LT88, have also been applied to the timed 
setting, namely in [KKLW07] to continuous-time Markov chains (IMC with no non-determinism), or in KKN09 to IMC. Nevertheless, these abstractions do not allow for constraints on time distributions. Instead they would employ abstractions on transition probabilities. Further, a compositional framework with timed specifications is presented in $\left[\mathrm{DLL}^{+} 12\right.$. This framework explicitly allows for time constraints. However, since the systems under consideration have nondeterministic flow of time (not stochastic), the natural choice was to only allow for discrete (not continuous) time constraints.

Although IMC support compositional design very well, analysis techniques for IMC proposed so far (e.g. $\mathrm{KZH}^{+} 11$ KKN09ZN10 GHKN12 are not compositional. They are all bound to the assumption that the analysed IMC is a closed system, i.e. it does not depend on interaction with the environment (all actions are internal). Some preliminary steps to develop a framework for synthesis of controllers based on models of hardware and control requirements have been taken in Mar11. The first attempt at compositionality is our very recent work $\left.\mathrm{BHK}^{+} 12\right]$ discussed above.

Algorithms for the time-bounded reachability problem for closed IMC have been given in [ZN10BS11HH13] and compositional abstraction techniques to compute it are developed in [KKN09. In the closed interpretation, IMC have some similarities with continuous-time Markov decision processes. For this formalism, algorithms for time-bounded reachability are developed in BHKH05 BS11.

\section{Interactive Markov Chains}

In this section, we introduce the formalism of interactive Markov chains together with the standard way to compose them. We denote by $\mathbb{N}, \mathbb{R}_{>0}$, and $\mathbb{R}_{>0}$ the sets of positive integers, positive real numbers and non-negative real numbers, respectively. Further, let $\mathcal{D}(S)$ denote the set of probability distributions over the set $S$.

Definition 1 (IMC). An interactive Markov chain (IMC) is a quintuple $\mathcal{C}=$ $\left(S, \mathbb{A c t}{ }^{\tau}, \hookrightarrow, \rightsquigarrow, s_{0}\right)$ where $S$ is a finite set of states, $\mathbb{A c t}^{\tau}$ is a finite set of actions containing a designated internal action $\tau, s_{0} \in S$ is an initial state,

$-\hookrightarrow \subseteq S \times \mathbb{A c t}^{\tau} \times S$ is an interactive transition relation, and

- $\subseteq S \times \mathbb{R}_{>0} \times S$ is a Markovian transition relation.

Elements of $\mathbb{A c t}:=\mathbb{A c t}^{\tau} \backslash\{\tau\}$ are called external actions. We write $s \stackrel{a}{\hookrightarrow} t$ whenever $(s, a, t) \in \hookrightarrow$, and $s \stackrel{\lambda}{\rightsquigarrow} t$ whenever $(s, \lambda, t) \in \rightsquigarrow$ where $\lambda$ is called a rate of the transition. We say that an external action $a$, or internal $\tau$, or Markovian transition is available in $s$, if $s \stackrel{a}{\hookrightarrow} t, s \stackrel{\tau}{\hookrightarrow} t$ or $s \stackrel{\lambda}{\rightsquigarrow} t$ for some $t$ (and $\lambda$ ), respectively.

IMC are well suited for compositional modelling, where systems are built out of smaller ones using standard composition operators. Parallel composition $\|_{A}$ over a synchronization alphabet $A$ produces a product of two IMC with transitions given by the rules

(PC1) $\left(s_{1}, s_{2}\right) \stackrel{a}{\hookrightarrow}\left(s_{1}^{\prime}, s_{2}^{\prime}\right)$ for each $s_{1} \stackrel{a}{\hookrightarrow} s_{1}^{\prime}$ and $s_{2} \stackrel{a}{\hookrightarrow} s_{2}^{\prime}$ and $a \in A$, (PC2, PC3) $\left(s_{1}, s_{2}\right) \stackrel{a}{\hookrightarrow}\left(s_{1}^{\prime}, s_{2}\right)$ for each $s_{1} \stackrel{a}{\hookrightarrow} s_{1}^{\prime}$ and $a \notin A$, and symetrically, 
(PC4, PC5) $\left(s_{1}, s_{2}\right) \stackrel{\lambda}{\rightsquigarrow}\left(s_{1}^{\prime}, s_{2}\right)$ for each $s_{1} \stackrel{\lambda}{\rightsquigarrow} s_{1}^{\prime}$, and symmetrically.

Further, hiding \A an alphabet $A$, yields a system, where each $s \stackrel{a}{\hookrightarrow} s^{\prime}$ with $a \notin A$ is left as it is, and each $s \stackrel{a}{\hookrightarrow} s^{\prime}$ with $a \in A$ is replaced by internal $s \stackrel{\tau}{\hookrightarrow} s^{\prime}$.

Hiding \Act thus yields a closed IMC, where external actions do not appear as transition labels (i.e. $\hookrightarrow \subseteq S \times\{\tau\} \times S$ ). A closed IMC (under a scheduler $\sigma$, see below) moves from state to state and thus produces a run which is an infinite sequence of the form $s_{0} t_{1} s_{1} t_{2} s_{2} \cdots$ where $s_{n}$ is the $n$-th visited state and $t_{n}$ is the time of arrival to $s_{n}$. After $n$ steps, the scheduler resolves the nondeterminism among internal $\tau$ transitions based on the path $\mathfrak{p}=s_{0} t_{1} \cdots t_{n} s_{n}$.

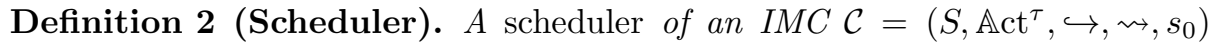
is a measurable function $\sigma:\left(S \times \mathbb{R}_{\geq 0}\right)^{*} \times S \rightarrow \mathcal{D}(S)$ such that for each path $\mathfrak{p}=s_{0} t_{1} s_{1} \cdots t_{n} s_{n}$ with $s_{n}$ having $\tau$ available, $\sigma(\mathfrak{p})(s)>0$ implies $s_{n} \stackrel{\tau}{\hookrightarrow} s$. The set of all schedulers for $\mathcal{C}$ is denoted by $\mathfrak{S}(\mathcal{C})$.

The decision of the scheduler $\sigma(\mathfrak{p})$ determines $t_{n+1}$ and $s_{n+1}$ as follows. If $s_{n}$ has available $\tau$, then the run proceeds immediately, i.e. at time $t_{n+1}:=t_{n}$, to a state $s_{n+1}$ randomly chosen according to the distribution $\sigma(\mathfrak{p})$. Otherwise, only Markovian transitions are available in $s_{n}$. In such a case, after waiting for a random time $t$ chosen according to the exponential distribution with the rate $\mathrm{R}\left(s_{n}\right)=\sum_{s_{n} \underset{\sim}{\prime}} \lambda$, the run moves at time $t_{n+1}:=t_{n}+t$ to a randomly chosen next state $s_{n+1}$ with probability $\lambda / r$ where $s_{n} \stackrel{\lambda}{\rightsquigarrow} s_{n+1}$. This defines a probability space ( $\mathbb{R u n s}, \mathcal{F}, \mathcal{P}_{\mathcal{C}}^{\sigma}$ ) over the runs in the standard way [ZN10].

\section{Time-Bounded Reachability}

In this section, we introduce the studied problems. One of the fundamental problems in verification and performance analysis of continuous-time stochastic systems is time-bounded reachability. Given a closed IMC $\mathcal{C}$, a set of goal states $G \subseteq S$ and a time bound $T \in \mathbb{R}_{\geq 0}$, the value of time-bounded reachability is defined as $\sup _{\sigma \in \mathfrak{S}(\mathcal{C})} \mathcal{P}_{\mathcal{C}}^{\sigma}\left[\diamond^{\leq T} G\right]$ where $\mathcal{P}_{\mathcal{C}}^{\sigma}\left[\diamond^{\leq T} G\right]$ denotes the probability that a run of $\mathcal{C}$ under the scheduler $\sigma$ visits a state of $G$ before time $T$. We have seen an example in the introduction. A standard assumption over all analysis techniques published for IMC $\mathrm{KZH}^{+} 11$ KKN09ZN10 GHKN12 is that each cycle contains a Markovian transition. It implies that the probability of taking infinitely many transitions in finite time, i.e. of Zeno behaviour, is zero. One can $\varepsilon$-approximate the value and compute the respective scheduler in time $\mathcal{O}\left(\lambda^{2} T^{2} / \varepsilon\right)$ [N10 recently improved to $\mathcal{O}\left(\sqrt{\lambda^{3} T^{3} / \varepsilon}\right)$ HH13.

For an open IMC to be put in parallel with an unknown environment, the optimal scheduler is computed so that it optimizes the guarantee against all possible environments. Formally, for an $\operatorname{IMC} \mathcal{C}=\left(C, \mathbb{A c t}^{\tau}, \hookrightarrow, \rightsquigarrow, c_{0}\right)$ and an environment IMC $\mathcal{E}$ with the same action alphabet $\mathbb{A c t}^{\tau}$, we introduce a composition $\mathcal{C} \mid \mathcal{E}=\left(\mathcal{C} \|_{\text {Act }} \mathcal{E}\right) \backslash \mathbb{A c t}$ where all open actions are hidden, yielding a closed system. In order to compute guarantees on $\mathcal{C} \mid \mathcal{E}$ provided we use a scheduler $\sigma$ in $\mathcal{C}$, we consider schedulers $\pi$ of $\mathcal{C} \mid \mathcal{E}$ that respect $\sigma$ on the internal actions of $\mathcal{C}$, written $\pi \in \mathfrak{S}_{\sigma}(\mathcal{C} \mid \mathcal{E})$; the formal definition is below. The value of compositional time-bounded reachability is then defined in $\left[\mathrm{BHK}^{+} 12\right]$ as 


$$
\sup _{\sigma \in \mathfrak{S}(\mathcal{C})} \inf _{\substack{\mathcal{E} \in \mathrm{ENV} \\ \pi \in \mathfrak{S}_{\sigma}(\mathcal{C} \mid \mathcal{E})}} \mathcal{P}_{\mathcal{C} \mid \mathcal{E}}^{\pi}\left[\diamond^{\leq T} G\right]
$$

where ENV denotes the set of all IMC with the action alphabet $\mathbb{A c t}^{\tau}$ and $\diamond^{\leq T} G$ is the set of runs that reach $G$ in the first component before $T$. Now $\pi$ respects $\sigma$ on internal actions of $\mathcal{C}$ if for every path $\mathfrak{p}=\left(c_{0}, e_{0}\right) t_{1} \cdots t_{n}\left(c_{n}, e_{n}\right)$ of $\mathcal{C} \mid \mathcal{E}$ there is $p \in[0,1]$ such that for each internal transition $c_{n} \stackrel{\tau}{\hookrightarrow} c$ of $\mathcal{C}$, we have $\pi(\mathfrak{p})\left(c, e_{n}\right)=p \cdot \sigma\left(\mathfrak{p}_{\mathcal{C}}\right)(c)$. Here $\mathfrak{p}_{\mathcal{C}}$ is the projection of $\mathfrak{p}$ where $\sigma$ can only see the path of moves in $\mathcal{C}$ and not in which states $\mathcal{E}$ is. Formally, we define observation of a path $\mathfrak{p}=\left(c_{0}, e_{0}\right) t_{1} \cdots t_{n}\left(c_{n}, e_{n}\right)$ as $\mathfrak{p}_{\mathcal{C}}=c_{0} t_{1} \cdots t_{n} c_{n}$ where each maximal consecutive sequence $t_{i} c_{i} \cdots t_{j} c_{j}$ with $c_{k}=c_{i}$ for all $i \leq k \leq j$ is rewritten to $t_{i} c_{i}$. This way, $\sigma$ ignores precisely the internal steps of $\mathcal{E}$.

\subsection{Specifications of environments}

In the second example in the introduction, without any assumptions on the environment only zero guarantees could be derived. The component was thus indistinguishable from an entirely useless one. In order to get a better guarantee, we introduce a formalism to specify assumptions on the behaviour of environments.

Example 1. In the mentioned example, if we knew that after an occurrence of req the environment is ready to synchronize on resp in time distributed according to $\operatorname{Exp}(3)$ or faster, we would be able to derive a guarantee of 0.26 . We will depict this assumption as shown below.

The dashed arrows denote may transitions, which may or may not be available, whereas the full arrows denote must transitions, which the environment is ready to synchronize on. Full arrows are further used for time transitions.

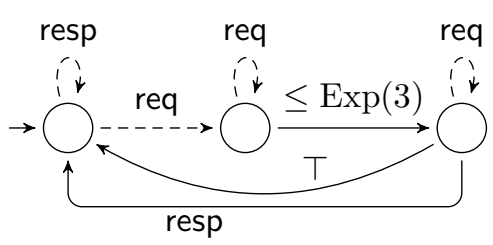

Although such a system resembles a timed automaton, there are several fundamental differences. Firstly, the time constraints are given by probability distributions instead of constants. Secondly, there is only one clock that, moreover, gets reset whenever the state is changed. Thirdly, we allow modalities of may and must transitions. Further, as usual with timed or stochastic specifications, we require determinism.

Definition 3 (MCA syntax). A continuous time constraint is either $\top$ or of the form $\bowtie d$ with $\bowtie \in\{\leq, \geq\}$ and $d$ a continuous distribution. We denote the set of all continuous time constraints by $\mathcal{C} \mathcal{T C}$. A modal continuous-time automaton (MCA) over $\Sigma$ is a tuple $\mathcal{S}=\left(Q, q_{0},--\rightarrow, \longrightarrow, \rightsquigarrow\right)$, where

- $Q$ is a non-empty finite set of locations and $q_{0} \in Q$ is an initial location,

$-\longrightarrow,-\rightarrow: Q \times \Sigma \rightarrow Q$ are must and may transition functions, respectively, satisfying $\longrightarrow \subseteq--\rightarrow$,

$-\rightsquigarrow: Q \rightarrow \mathcal{C T C} \times Q$ is a time flow function. 
We have seen an example of an MCA in the previous example. Note that upon taking req from the first state, the waiting time is chosen and the waiting starts. On the other hand, when req self-loop is taken in the middle state, the waiting process is not restarted, but continues on the background independently (1) We introduce this independence as a useful feature to model properties as "response follows within some time after request" in the setting with concurrently running processes. Further, we have transitions under $T$ corresponding to " $>0$ ", meaning there is no restriction on the time distribution except that the transition takes non-zero time. We formalize this in the following definition. With other respects, the semantics of may and must transitions follows the standards of modal transition systems [LT88.

Definition 4 (MCA semantics). An IMC $\mathcal{E}=\left(E, \mathbb{A c t}^{\tau}, \hookrightarrow, \rightsquigarrow, e_{0}\right)$ conforms to an $M C A$ specification $\mathcal{S}=\left(Q, q_{0},-\rightarrow, \longrightarrow, \rightsquigarrow\right)$, written $\mathcal{E} \models \mathcal{S}$, if there is a satisfaction relation $\mathcal{R} \subseteq E \times Q$ containing $\left(e_{0}, q_{0}\right)$ and satisfying for each $(e, q) \in \mathcal{R}$ that whenever

1. $q \stackrel{a}{\longrightarrow} q^{\prime}$ then there is some $e \stackrel{a}{\hookrightarrow} e^{\prime}$ and if, moreover, $q \neq q^{\prime}$ then $e^{\prime} \mathcal{R} q^{\prime}$,

2. $e^{\stackrel{a}{\hookrightarrow}} e^{\prime}$ then there is (unique) $q^{\stackrel{a}{\rightarrow}} q^{\prime}$ and if, moreover, $q \neq q^{\prime}$ then $e^{\prime} \mathcal{R} q^{\prime}$,

3. $e^{\stackrel{\tau}{\rightarrow}} e^{\prime}$ then $e^{\prime} \mathcal{R} q$,

4. $q \stackrel{c t c}{\rightsquigarrow} q^{\prime}$ then for every IMC $\mathcal{C}$ and every scheduler $\pi \in \mathfrak{S}(\mathcal{C} \mid e)$ there is a random variable Stop $: \mathbb{R u n s} \rightarrow \mathbb{R}_{>0}$ on the probability space $\left(\mathbb{R u n s}, \mathcal{F}, \mathcal{P}_{\mathcal{C} \mid e}^{\pi}\right)$ such that

- if ctc is of the form $\bowtie d$ then the cumulative distribution function of Stop is point-wise $\bowtie$ cumulative distribution function of $d$ (there are no constraints when ctc $=\top$ ), and

- for every run $\rho$ of $\mathcal{C} \mid e$ under $\pi$, either a transition corresponding to synchronization on action a with $q_{\stackrel{a}{-} \rightarrow}^{\rightarrow} q^{\prime \prime} \neq q$ is taken before time Stop $(\rho)$, or

- the state $\left(c, e^{\prime}\right)$ visited at time Stop $(\rho)$ satisfies $e^{\prime} \mathcal{R} q^{\prime}$, and

- for all states $(\bar{c}, \bar{e})$ visited prior to that, whenever

(a) $q \stackrel{a}{\longrightarrow} q^{\prime \prime}$ then there is $\bar{e} \stackrel{a}{\hookrightarrow} e^{\prime}$,

(b) $\bar{e} \stackrel{a}{\hookrightarrow} e^{\prime}$ then there is $q^{-\rightarrow} \rightarrow q^{\prime \prime}$.

The semantics of $\mathcal{S}$ is the set $\llbracket \mathcal{S} \rrbracket=\{\mathcal{E} \in \mathrm{IMC} \mid \mathcal{E} \models \mathcal{S}\}$ of all conforming IMC.

Example 2. We illustrate this definition. Consider the MCA on the right above specifying that $a$ is ready and $b$ will be ready either immediately after taking $a$ or within the time distributed according to the Erlang distribution $\operatorname{Er}(3,1)$, which is a convolution of three $\operatorname{Exp}(1)$ distributions. The IMC below conforms to this specification (here, Stop $\sim \operatorname{Er}(2,1)$ can be chosen).

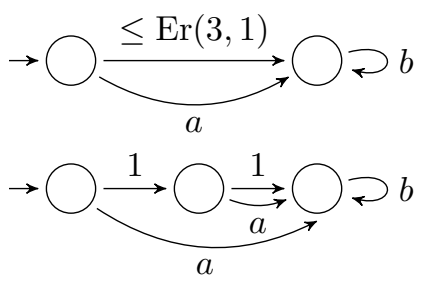

(1) This makes no difference for memoryless exponential distributions, but for all other distributions it does.

(2) Here $e$ stands for the IMC $\mathcal{E}$ with the initial state $e$. 
However, observe that it would not conform, if there was no transition under $a$ from the middle to the right state. Satisfying the modalities throughout the waiting is namely required by the last bullet of the previous definition.

\subsection{Assume-Guarantee Optimization}

We can now formally state what guarantees on time-bounded reachability we can derive provided the unknown environment conforms to a specification $\mathcal{S}$. Given an open IMC $\mathcal{C}$, a set of goal states $G \subseteq C$ and a time bound $T \in \mathbb{R}_{\geq 0}$, the value of compositional time-bounded reachability conditioned by an $M C A \mathcal{S}$ is defined as

$$
v_{\mathcal{S}}(\mathcal{C}):=\sup _{\sigma \in \mathfrak{S}(\mathcal{C})} \inf _{\substack{\mathcal{E} \in \operatorname{ENV}: \mathcal{E} \mid=\mathcal{S} \\ \pi \in \mathfrak{S}_{\sigma}(\mathcal{C} \mid \mathcal{E})}} \mathcal{P}_{\mathcal{C} \mid \mathcal{E}}^{\pi}\left[\diamond^{T} G\right]
$$

In this paper, we pose a technical assumption on the set of schedulers of $\mathcal{C}$. For some clock resolution $\delta>0$, we consider only such schedulers $\sigma$ that take the same decision for any pair of paths $c_{0} t_{1} \ldots t_{n} c_{n}$ and $c_{0} t_{1}^{\prime} \ldots t_{n}^{\prime} c_{n}$ with $t_{i}$ and $t_{i}^{\prime}$ equal when rounded down to a multiple of $\delta$ for all $1 \leq i \leq n$. This is no practical restriction as it is not possible to achieve arbitrary resolution of clocks when implementing the scheduler. Observe this is a safe assumption as it is not imposed on the unknown environment.

We consider specifications $\mathcal{S}$ where distributions have differentiable density functions. In the rest of the paper we show how to approximate $v_{\mathcal{S}}(\mathcal{C})$ for such $\mathcal{S}$. Firstly, we make a product of the given IMC and MCA. Secondly, we transform the product to a game. This game is further discretized into a partially observable stochastic game played on a dag where the quantitative reachability is solved. For full proofs, see the appendix.

\section{Product of IMC and Specification}

In this section, we first translate MCA $\mathcal{S}$ into a sequence of IMC $\left(\mathcal{S}_{i}\right)_{i \in \mathbb{N}}$. Second, we combine the given IMC $\mathcal{C}$ with the sequence $\left(\mathcal{S}_{i}\right)_{i \in \mathbb{N}}$ into a sequence of product IMC $\left(\mathcal{C} \times \mathcal{S}_{i}\right)_{i \in \mathbb{N}}$ that will be further analysed. The goal is to reduce the case where the unknown environment is bound by the specification to a setting where we solve the problem for the product IMC while quantifying over all possible environments (satisfying only a simple technical assumption discussed at the end of the section), denoted ENV'. The reason why we need a sequence of products instead of one product is that we need to approximate arbitrary distributions with more and more precise and detailed hyper-Erlang distributions expressible in IMC. Formally, we want to define the sequence of the products $\mathcal{C} \times \mathcal{S}_{i}$ so that

$$
v_{\text {product }}\left(\mathcal{C} \times \mathcal{S}_{i}\right) \quad:=\sup _{\sigma \in \mathfrak{S}(\mathcal{C})} \inf _{\substack{\left.\mathcal{E} \in \mathrm{ENV}^{\prime} \\ \pi \in \mathfrak{S}_{\sigma}\left(\mathcal{C} \times \mathcal{S}_{i}\right) \mid \mathcal{E}\right)}} \mathcal{P}_{\left(\mathcal{C} \times \mathcal{S}_{i}\right) \mid \mathcal{E}}^{\pi}\left[\diamond^{\leq T} G\right]
$$

approximates the compositional value:

Theorem 1. For every $I M C \mathcal{C}$ and $M C A \mathcal{S}, v_{\mathcal{S}}(\mathcal{C})=\lim _{i \rightarrow \infty} v_{\text {product }}\left(\mathcal{C} \times \mathcal{S}_{i}\right)$. 
Note that in $v_{\text {product }}, \sigma$ is a scheduler over $\mathcal{C}$, not the whole product $\mathcal{C} \times \mathcal{S}_{i}$ (3) Constructing a product with the specification intuitively corresponds to adding a known, but uncontrollable and unobservable part of the environment to $\mathcal{C}$. We proceed as follows: We translate the MCA $\mathcal{S}$ into a sequence of IMC $\mathcal{S}_{i}$ and then the product will be defined as basically a parallel composition of $\mathcal{C}$ and $\mathcal{S}_{i}$.

There are two steps in the translation of $\mathcal{S}$ to $\mathcal{S}_{i}$. Firstly, we deal with the modal transitions. A may transition under $a$ is translated to a standard external transition under $a$ that has to synchronize with $a$ in both $\mathcal{C}$ and $\mathcal{E}$ simultaneously, so that the environment may or may not let the synchronization occur. Further, each must transition under $a$ is replaced by an external transition, that synchronizes with $a$ in $\mathcal{C}$, but is hidden before making product with the environment. This way, we guarantee that $\mathcal{C}$ can take $a$ and make progress no matter if the general environment $\mathcal{E}$ would like to synchronize on $a$ or not.

Formally, the must transitions are transformed into special "barred" transitions that will be immediately hidden in the product $\mathcal{C} \times \mathcal{S}_{i}$ as opposed to transitions arising from may transitions. Let $\overline{\mathbb{A} c t}=\{\bar{a} \mid a \in \mathbb{A} c t\}$ denote a fresh copy of the original alphabet. We replace all modal transitions as follows

- whenever $q \stackrel{a}{-\rightarrow} r$ set $q \stackrel{a}{\hookrightarrow} r$,

- whenever $q \stackrel{a}{\longrightarrow} r$ set $q \stackrel{\vec{a}}{\hookrightarrow} r$.

The second step is to deal with the timed transitions, especially with the constraints of the form $\bowtie d$. Such a transition is, roughly speaking, replaced by a phase-type approximation of $d$. This is a continuous-time Markov chain (an IMC with only timed transitions) with a sink state such that the time to reach the sink state is distributed with $d^{\prime}$. For any continuous distribution $d$, we can find such $d^{\prime}$ arbitrarily close to $d$.

Example 3. Consider the following MCA on the left. It specifies that whenever ask is taken, it cannot be taken again for at least the time distributed by $\operatorname{Er}(2, \lambda)$ and during all that time, it is ready to synchronize on answer. This specifies systems that are allowed to ask, but not too often, and whenever they ask, they must be ready to receive (possibly more) answers for at least the specified time.

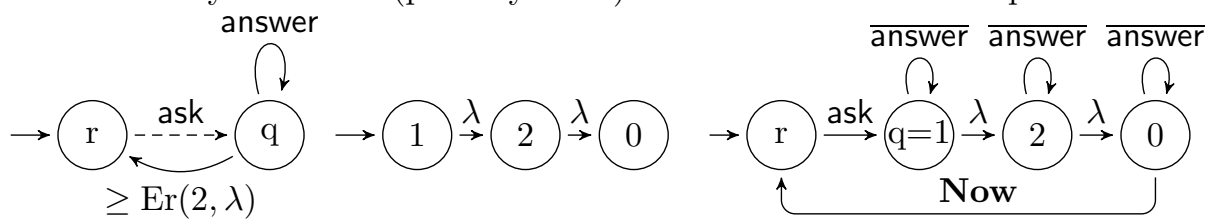

After performing the first step of replacing the modal transitions as described above, we proceed with the second step as follows. We replace the timed transition with a phase-type, e.g. the one represented by the IMC in the middle. Observe that while the Markovian transitions are taken, answer must still be available. Hence, we duplicate the corresponding self-loops on all the new states. Further, since the time constraint is of the form $\geq$, getting to the state $(q, 0)$

(3) Here we overload the notation $\mathfrak{S}_{\sigma}\left(\left(\mathcal{C} \times \mathcal{S}_{i}\right) \mid \mathcal{E}\right)$ introduced for pairs in a straightforward way to triples, where $\sigma$ ignores both the second and the third components. 
does not guarantee that we already get to the state $r$. It can possibly take longer. To this end, we connect the states $(q, 0)$ and $r$ by a special external action Now. Since this action is synchronized with $\mathcal{E} \in \mathrm{ENV}^{\prime}$, the environment can block the progress for arbitrarily long time. Altogether, we obtain the IMC on the right.

In the case of " $\leq$ " condition, we would instead add the Now transition from each auxiliary state to the sink, which could instead shorten the waiting time.

When constructing $\mathcal{S}_{i}$, we replace each distribution $d$ with its hyper-Erlang phase-type approximation $d_{i}$ with $i$ branches of lengths 1 to $i$ and rates $\sqrt{i}$ in each branch. For formal description, see Appendix A.2. Formally, let Now $\notin \mathbb{A}$ ct $\cup \overline{\mathbb{A} c t}$ be a fresh action. We replace all timed transitions as follows:

- whenever $q \stackrel{\top}{\rightsquigarrow} r$ such that $q \neq r$ set $q \stackrel{\text { Now }}{\hookrightarrow} r$,

- whenever $q \stackrel{\bowtie d}{\rightsquigarrow}$ where the phase-type $d_{i}$ corresponds to a continuous-time Markov chain (IMC with only timed transitions) with the set of states $D$, the initial state 1 and the sink state 0 , then

1. identify the states $q$ and 1 ,

2. for every $u \in D$ and $q \stackrel{\alpha}{\hookrightarrow} q$, set $u \stackrel{\alpha}{\hookrightarrow} u$,

3. for every $u \in D$ and $q \stackrel{\alpha}{\hookrightarrow} p$ with $p \neq q$, set $\underset{\text { Now }}{\stackrel{\alpha}{\leftrightarrow}} p$,

4. if $\bowtie=\leq$, then identify $r$ and 0 , and set $u \stackrel{\text { Now }}{\hookrightarrow} r$ for each $u \in D$,

5. if $\bowtie=\geq$, then set $0 \stackrel{\text { Now }}{\hookrightarrow} r$.

Intuitively, the new timed transitions model the delays, while in the " $\leq$ " case, the action Now can be taken to speed up the process of waiting, and in the " $\geq$ " case, Now can be used to block further progress even after the delay has elapsed.

The product is now the parallel composition of $\mathcal{C}$ and $\mathcal{S}_{i}$, where each action $\bar{a}$ synchronizes with $a$ and the result is immediately hidden. Formally, the product $\mathcal{C} \times \mathcal{S}$ is defined as $\mathcal{C} \|_{\mathbb{A c t} \cup \overline{\mathbb{A c t}}}^{\mathbf{P C 6}} \mathcal{S}_{i}$, where $\|_{\mathbb{A c t} \cup \overline{\mathbb{A c t}}}^{\mathbf{P C 6}}$ is the parallel composition with one additional axiom:

(PC6) $s_{1} \stackrel{a}{\hookrightarrow} s_{1}^{\prime}$ and $s_{2} \stackrel{\bar{a}}{\hookrightarrow} s_{2}^{\prime}$ implies $\left(s_{1}, s_{2}\right) \stackrel{\tau}{\hookrightarrow}\left(s_{1}^{\prime}, s_{2}^{\prime}\right)$,

saying that $a$ synchronizes also with $\bar{a}$ and, in that case, is immediately hidden (and any unused $\bar{a}$ transitions are thrown away).

The idea of Now is that it can be taken in arbitrarily short, but non-zero time. To this end, we define $\mathrm{ENV}^{\prime}$ in the definition of $v_{\text {product }}\left(\mathcal{C} \times \mathcal{S}_{i}\right)$ to denote all environments where Now is only available in states that can be entered by only a Markovian transition. Due to this requirement, each Now can only be taken after waiting for some time.

\section{Controller-Environment Games}

So far, we have reduced our problem to computing $\lim _{i \rightarrow \infty} v_{\text {product }}\left(\mathcal{C} \times \mathcal{S}_{i}\right)$. Note that we are still quantifying over unknown environments. Further, the behaviour of each environment is limited by the uncontrollable stochastic flow of time caused by its Markovian transitions. This setting is still too difficult to be solved directly. Therefore, in this section, we reduce this setting to one, where 
the stochastic flow of time of the environment (limited in an unknown way) is replaced by a free non-deterministic choice of the second player.

We want to turn the product IMC $\mathcal{C} \times \mathcal{S}_{i}$ into a two-player controllerenvironment game (CE game) $\mathcal{G}_{i}$, where player con controls the decisions over internal transitions in $\mathcal{C}$; and player env simulates the environment including speeding-up/slowing-down $\mathcal{S}$ using Now transitions. In essence, con chooses in each state with internal transitions one of them, and env chooses in each state with external (and hence synchronizing) transitions either which of them should be taken, or a delay $d \in \mathbb{R}_{>0}$ during which no synchronization occurs. The internal and external transitions take zero time to be executed if chosen. Otherwise, the game waits until either the delay $d$ elapses or a Markovian transition occurs.

This is the approach taken in $\mathrm{BHK}^{+} 12$ where no specification is considered. However, there is a catch. This construction is only correct under the assumption of $\left[\mathrm{BHK}^{+} 12\right]$ that there are no states of $\mathcal{C}$ with both external and internal transitions available.

Example 4. Consider the IMC $\mathcal{C}$ on the right (for instance with a trivial specification not restricting the environment). Note that there are both internal and external actions available in no.

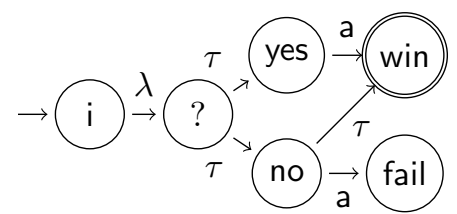

As $\tau$ transitions take zero time, the environment $\mathcal{E}$ must spend almost all the time in states without $\tau$. Hence, when ? is entered, $\mathcal{E}$ is almost surely in such a state $e$. Now $\tau$ form ? is taken and $\mathcal{E}$ cannot move to another state when yes/no is entered. Since action a either is or is not available in $e$, the environment cannot choose to synchronize in no and not to synchronize in yes. As a result, the environment "commits" in advance to synchronize over a either in both yes and no or in none of them. Therefore, in the game we define, env cannot completely freely choose which external transition is/is not taken. Further, note that the scheduler of $\mathcal{C}$ cannot observe whether a is currently available in $\mathcal{E}$, which intrinsically induces imperfect information.

In order to transfer these "commitments" to the game, we again make use of the compositionality of IMC and put the product $\mathcal{C} \times \mathcal{S}_{i}$ in parallel with an IMC Commit and then define the game on the result.

The action alphabet of Commit is $\mathbb{A c t} \cup$ $\{$ Now, Change $\}$ and the state space is $2^{\text {Act }} \cup$ $\{$ commit, now? $\}$ (in the figure, Act $=\{\mathrm{a}\}$; for formal description, see Appendix A.3. State $A \subseteq$ Act corresponds to $\mathcal{E}$ being committed to the set of currently available actions $A$. Thus

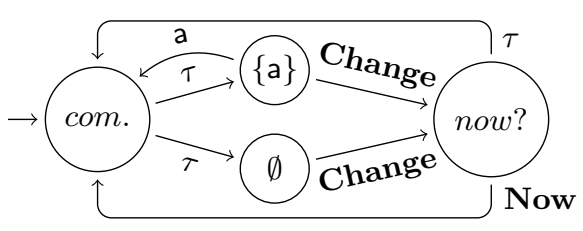
$A \stackrel{a}{\hookrightarrow}$ commit for each $a \in A$. This commitment must be respected until the state of $\mathcal{E}$ is changed: either (1) by an external transition from the commitment set (which in Commit leads to the state commit where a new commitment is immediately chosen); or (2) by a Change transition (indicating the environment changed its state due to its Markovian transition).

The game $\mathcal{G}_{i}$ is played on the arena $\left(\mathcal{C} \times \mathcal{S}_{i} \|_{\mathbb{A c t} \cup\{\text { Now }\}}\right.$ Commit $) \backslash(\mathbb{A c t} \cup\{$ Now $\})$ with its set of states denoted by $G_{i}$. Observe that external actions have either 
been hidden (whenever they were available in the commitment), or discarded (whenever not present in the current commitment). The only external action that remains is Change. The game $\mathcal{G}_{i}$ is played as follows. There are two types of states: immediate states with some $\tau$ transitions available and timed states with no $\tau$ available. The game starts in $v_{0}=\left(c_{0}, q_{0}\right.$, commit $)$.

- In an immediate state $v_{n}=(c, q, e)$, con chooses a probability distribution over transitions corresponding to the internal transitions in $\mathcal{C}$ (if there are any). Then, env either approves this choice (chooses $\checkmark$ ) and $v_{n+1}$ is chosen randomly according to this distribution, or rejects this choice and chooses a $\tau$ transition to some $v_{n+1}$ such that the transition does not correspond to any internal transitions of $\mathcal{C}$. Then the game moves at time $t_{n+1}=t_{n}$ to $v_{n+1}$.

- In a timed state $v_{n}=(c, q, e)$, env chooses a delay $d>0$. Then Markovian transitions (if available) are resolved by randomly sampling a time $t$ according to the exponential distribution with rate $\mathrm{R}\left(v_{n}\right)$ and randomly choosing a target state $v_{n+1}$ where each $v_{n} \stackrel{\lambda}{\rightsquigarrow} v$ is chosen with probability $\lambda / \mathrm{R}\left(v_{n}\right)$.

- If $t<d, \mathcal{G}_{i}$ moves at time $t_{n+1}=t_{n}+t$ to $v_{n+1}$, (Markovian transition wins)

- else $\mathcal{G}_{i}$ moves at time $t_{n+1}=t_{n}+d$ to $(c, q$, now? $) . \quad(\mathcal{E}$ takes Change $)$

This generates a run $v_{0} t_{1} v_{1} t_{1} \cdots$. The set $\left(G_{i} \times \mathbb{R}_{\geq 0}\right)^{*} \times G_{i}$ of prefixes of runs is denoted Histories $(\mathcal{G})$. We formalize the choice of con as a strategy $\sigma: \mathbb{H}$ istories $\left(\mathcal{G}_{i}\right) \rightarrow \mathcal{D}\left(G_{i}\right)$. We further allow the env to randomize and thus his strategy is $\pi: \mathbb{H}$ istories $\left(\mathcal{G}_{i}\right) \rightarrow \mathcal{D}\left(\{\checkmark\} \cup G_{i}\right) \cup \mathcal{D}\left(\mathbb{R}_{>0}\right)$. We denote by $\Sigma$ and $\Pi$ the sets of all strategies of the players con and env, respectively.

Since con is not supposed to observe the state of the specification and the state of Commit, we consider in $\Sigma$ only those strategies that satisfy $\sigma(p)=$ $\sigma\left(p^{\prime}\right)$, whenever observations of $p$ and $p^{\prime}$ are the same. Like before, the observation of $\left(c_{0}, q_{0}, e_{0}\right) t_{1} \cdots t_{n}\left(c_{n}, q_{n}, e_{n}\right) \in \mathbb{H}$ istories $(\mathcal{G})$ is a sequence obtained from $c_{0} t_{1} \cdots t_{n} c_{n}$ by replacing each maximal consecutive sequence $t_{i} c_{i} \cdots t_{j} c_{j}$ with all $c_{k}$ the same, by $t_{i} c_{i}$. This replacement takes place so that the player cannot observe transitions that do not affect $\mathcal{C}$. Notice that now $\mathfrak{S}(\mathcal{C})$ is in one-to-one correspondence with $\Sigma$. Further, in order to keep CE games out of Zeno behaviour, we consider in $\Pi$ only those strategies for which the induced Zeno runs have zero measure, i.e. the sum of the chosen delays diverges almost surely no matter what con is doing. The value of $\mathcal{G}_{i}$ is now defined as

$$
v_{\mathcal{G}_{i}}:=\sup _{\sigma \in \Sigma} \inf _{\pi \in \Pi} \mathcal{P}_{\mathcal{G}_{i}}^{\sigma, \pi}\left[\diamond^{\leq T} G\right]
$$

where $\mathcal{P}_{\mathcal{G}_{i}}^{\sigma, \pi}\left[\diamond^{\leq T} G\right]$ is the probability of all runs of $\mathcal{G}_{i}$ induced by $\sigma$ and $\pi$ and reaching a state with the first component in $G$ before time $T$. We now show that it coincides with the value of the $i$ th product:

Theorem 2. For every $I M C \mathcal{C}, M C A \mathcal{S}, i \in \mathbb{N}$, we have $v_{\mathcal{G}_{i}}=v_{\text {product }}\left(\mathcal{C} \times \mathcal{S}_{i}\right)$.

This result allows for approximating $v_{\mathcal{S}}(\mathcal{C})$ through computing $v_{\mathcal{G}_{i}}$ 's. However, from the algorithmic point of view, we would prefer approximating $v_{\mathcal{S}}(\mathcal{C})$ 
by solving a single game $\mathcal{G}$ whose value $v_{\mathcal{G}}$ we could approximate directly. This is indeed possible. But first, we need to clarify, why the approximation sequence $\mathcal{S}_{i}$ was crucial even in the case where all distributions of $\mathcal{S}$ are already exponential.

Consider the MCA on the right and a conforming environment $\mathcal{E}$, in which $a$ is available iff $b$ becomes available within 0.3 time units. If Player env wants to simulate this behaviour, he needs to know how long the transition to $r$ is

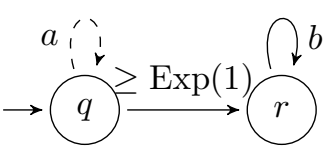
going to take so that he can plan his behaviour freely, only sticking to satisfying the specification. If we translate $\operatorname{Exp}(1)$ directly to a single Markovian transition (with no error incurred), env knows nothing about this time as exponential distributions are memoryless. On the other hand, with finer hyper-Erlang, he knows how long the current branch of hyper-Erlang is roughly going to take. In the limit, he knows the precise waiting time right after coming to $q$.

To summarize, env is too weak in $\mathcal{G}_{i}$, because it lacks the information about the precise time progress of the specification. The environment needs to know how much time is left before changing the location of $\mathcal{S}$. Therefore, the game $\mathcal{G}$ is constructed from $\mathcal{G}_{1}$ by multiplying the state space with $\mathbb{R}_{\geq 0}$ where we store the exact time to be waited. After the product changes the state so that the specification component switches to a state with $\bowtie d$ constraint, this last component is overwritten with a number generated according to $d$. This way, the environment knows precisely how much time is left in the current specification location. This corresponds to the infinitely precise hyper-Erlang, where we at the beginning randomly enter a particular branch, which is left in time with Dirac distribution. For more details, see Appendix $\mathrm{D}$.

Denoting the value of $\mathcal{G}$ by $v_{\mathcal{G}}:=\sup _{\sigma \in \Sigma} \inf _{\pi \in \Pi} \mathcal{P}_{\mathcal{G}}^{\sigma, \pi}\left[\diamond^{\leq T} G\right]$, we obtain:

Theorem 3. For every $I M C \mathcal{C}$ and $M C A \mathcal{S}$, we have $v_{\mathcal{G}}=\lim _{i \rightarrow \infty} v_{\mathcal{G}_{i}}$.

\section{Approximation using discrete-time $\mathrm{PO}$ games}

In this section, we briefly discuss the approximation of $v_{\mathcal{G}}$ by a discrete time turn-based partial-observation stochastic game $\Delta$. The construction is rather standard; hence, we do not treat the technical difficulties in great detail (see Appendix E). We divide the time bound $T$ into $N$ intervals of length $\kappa=T / N$ such that the clock resolution $\delta$ (see Section 3.2 ) satisfies $\delta=n \kappa$ for some $n \in \mathbb{N}$.

1. We enhance the state space with a counter $i \in\{0, \ldots, N\}$ that tracks that $i \cdot \kappa$ time has already elapsed. Similarly, the $\mathbb{R}_{\geq 0}$-component of the state space is discretized to $\kappa$-multiples. In timed states, time is assumed to pass exactly by $\kappa$. In immediate states, actions are assumed to take zero time.

2. We let at most one Markovian transition occur in one step in a timed state.

3. We unfold the game into a tree until on each branch a timed state with $i=N$ is reached. Thereafter, $\Delta$ stops. We obtain a graph of size bounded by $b \leq N \cdot|G|$ where $b$ is the maximal branching and $G$ is the state space of $\mathcal{G}$.

Let $\Sigma_{\Delta}$ and $\Pi_{\Delta}$ denote the set of randomized history-dependent strategies of con and env, respectively, where player con observes in the history only the 
first components of the states, i.e. the states of $\mathcal{C}$, and the elapsed time $\lfloor i / n\rfloor$ up to the precision $\delta$. Then $v_{\Delta}:=\sup _{\sigma \in \Sigma_{\Delta}} \inf _{\pi \in \Pi_{\Delta}} \mathcal{P}_{\Delta}^{\sigma, \pi}(\diamond G)$ denotes the value of the game $\Delta$ where $\mathcal{P}_{\Delta}^{\sigma, \pi}(\nabla G)$ is the probability of the runs of $\Delta$ induced by $\sigma$ and $\pi$ and reaching a state with first component in $G$. Let $b$ be a constant bounding (a) the sum of outgoing rates for any state of $\mathcal{C}$, and (b) densities and their first derivative for any distribution in $\mathcal{S}$.

Theorem 4. For every $I M C \mathcal{C}$ and $M C A \mathcal{S}, v_{\mathcal{G}}$ is approximated by $v_{\Delta}$ :

$$
\left|v_{\mathcal{G}}-v_{\Delta}\right| \leq 10 \kappa(b T)^{2} \ln \frac{1}{\kappa} .
$$

A strategy $\sigma^{*}$ optimal in $\Delta$ defines a strategy $\left(10 \kappa(b T)^{2} \ln \frac{1}{\kappa}\right)$-optimal in $\mathcal{G}$. Further, $v_{\Delta}$ and $\sigma^{*}$ can be computed in time polynomial in $|\Delta|$, hence in time $2^{\mathcal{O}(|\mathcal{G}|)}$.

The proof of the error bound extends the technique of the previous bounds of $\left[\mathrm{ZN10}\right.$ and $\left.\mathrm{BHK}^{+} 12\right]$. Its technical difficulty stems from partial observation and from semi-Markov behaviour caused by the arbitrary distributions in the specification. The game is unfolded into a tree in order to use the result of KMvS94. Without the unfolding, the best known (naive) solution would be a reduction to the theory of reals, yielding an EXPSPACE algorithm.

\section{Summary}

We have introduced an assume-guarantee framework for IMC. We have considered the problem to approximate the guarantee on time-bounded reachability properties in an unknown environment $\mathcal{E}$ that satisfies a given assumption. The assumptions are expressed in a new formalism, which introduces continuous time constraints. The algorithmic solution results from Theorems 1 to 4:

Corollary 1. For every IMCC and $M C A \mathcal{S}$ and $\varepsilon>0$, a value $v$ and a scheduler $\sigma$ can be computed in exponential time such that $\left|v_{\mathcal{S}}(\mathcal{C})-v\right| \leq \varepsilon$ and $\sigma$ is $\varepsilon$ optimal in $v_{\mathcal{S}}(\mathcal{C})$.

In future work, we want to focus on identifying structural subclasses of IMC allowing for polynomial analysis.

Acknowledgement The work has received support from the Czech Science Foundation, project No. P202/12/G061, from the German Science Foundation DFG as part of SFB/TR 14 AVACS, and from the EU FP7 Programme under grant agreement no. 295261 (MEALS) and 318490 (SENSATION). We also thank Tomáš Brázdil and Vojtěch Řehák for fruitful discussions and for their feedback.

\section{References}

AH96. R. Alur and T.A. Henzinger. Reactive modules. In LICS, pages 207-218, 1996.

BF09. P. Bouyer and V. Forejt. Reachability in stochastic timed games. In Proc. of ICALP, volume 5556 of $L N C S$, pages 103-114. Springer, 2009. 
$\mathrm{BHK}^{+} 12$. T. Brázdil, H. Hermanns, J. Krčál, J. Křetínský, and V. Řehák. Verification of open interactive markov chains. In FSTTCS, pages 474-485, 2012.

BHKH05. C. Baier, H. Hermanns, J.-P. Katoen, and B.R. Haverkort. Efficient computation of time-bounded reachability probabilities in uniform continuoustime Markov decision processes. Theor. Comp. Sci., 345(1):2-26, 2005.

BS11. P. Buchholz and I. Schulz. Numerical Analysis of Continuous Time Markov Decision processes over Finite Horizons. Computers and Operations Research, 38:651-659, 2011.

CD10. K. Chatterjee and L. Doyen. The complexity of partial-observation parity games. In LPAR (Yogyakarta), pages 1-14, 2010.

DLL $^{+}$12. A. David, K.G. Larsen, A. Legay, M.H. Møller, U. Nyman, A.P. Ravn, A. Skou, and A. Wasowski. Compositional verification of real-time systems using ECDAR. STTT, 14(6):703-720, 2012.

$\mathrm{EKN}^{+}$12. M.-A. Esteve, J.-P. Katoen, V.Y. Nguyen, B. Postma, and Y. Yushtein. Formal correctness, safety, dependability and performance analysis of a satellite. In Proc. of ICSE. ACM and IEEE press, 2012.

GHKN12. D. Guck, T. Han, J.-P. Katoen, and M.R. Neuhäußer. Quantitative timed analysis of interactive Markov chains. In NFM, volume 7226 of $L N C S$, pages 8-23. Springer, 2012.

HH13. H. Hatefi and H. Hermanns. Improving time bounded computations in interactive Markov chain. In FSEN, 2013. to appear.

HK09. H. Hermanns and J.-P. Katoen. The how and why of interactive Markov chains. In FMCO, volume 6286 of $L N C S$, pages 311-337. Springer, 2009.

$\mathrm{HKR}^{+}$10. B.R. Haverkort, M. Kuntz, A. Remke, S. Roolvink, and M.I.A. Stoelinga. Evaluating repair strategies for a water-treatment facility using Arcade. In Proc. of DSN, pages 419-424, 2010.

HMP01. T.A. Henzinger, M. Minea, and V.S. Prabhu. Assume-guarantee reasoning for hierarchical hybrid systems. In $H S C C$, pages 275-290, 2001.

$\mathrm{HNP}^{+}$11. E.M. Hahn, G. Norman, D. Parker, B. Wachter, and L. Zhang. Gamebased abstraction and controller synthesis for probabilistic hybrid systems. In QEST, pages 69-78, 2011.

KKLW07. J.-P. Katoen, D. Klink, M. Leucker, and V. Wolf. Three-valued abstraction for continuous-time Markov chains. In $C A V$, pages 311-324, 2007.

KKN09. J.-P. Katoen, D. Klink, and M.R. Neuhäußer. Compositional abstraction for stochastic systems. In FORMATS, pages 195-211, 2009.

KMvS94. D. Koller, N. Megiddo, and B. von Stengel. Fast algorithms for finding randomized strategies in game trees. In STOC, pages 750-759, 1994.

KV96. O. Kupferman and M. Vardi. Module checking. In $C A V$, volume 1102 of LNCS, pages 75-86. Springer, 1996.

KZH $^{+}$11. J.-P. Katoen, I.S. Zapreev, E.M. Hahn, H. Hermanns, and D.N. Jansen. The ins and outs of the probabilistic model checker MRMC. Performance Evaluation, 68(2):90-104, 2011.

LT88. K. G. Larsen and B. Thomsen. A modal process logic. In LICS, pages 203-210, 1988.

Mar11. J. Markovski. Towards supervisory control of interactive Markov chains: Controllability. In ACSD, pages 108-117, 2011.

MC81. J. Misra and K. Mani Chandy. Proofs of networks of processes. IEEE Trans. Software Eng., 7(4):417-426, 1981.

M.R10. M.R. Neuhäußer. Model checking nondeterministic and randomly timed systems. PhD thesis, University of Twente, 2010. 
RW89. P.J.G. Ramadge and W.M. Wonham. The control of discrete event systems. Proceedings of the IEEE, 77(1), 1989.

Spr11. J. Sproston. Discrete-time verification and control for probabilistic rectangular hybrid automata. In QEST, pages 79-88, 2011.

TAKB96. S. Tasiran, R. Alur, R.P. Kurshan, and R.K. Brayton. Verifying abstractions of timed systems. In CONCUR, pages 546-562, 1996.

ZN10. L. Zhang and M.R. Neuhäußer. Model checking interactive Markov chains. In Proc. of TACAS, volume 6015 of $L N C S$, pages 53-68. Springer, 2010. 


\section{Appendix}

\section{A Additional Examples and Technical Definitions}

\section{A.1 Product: An Example}

Example 5. Let us illustrate the product of the MCA from Example 3 and the IMC below. The MCA specifies a client who might want to ask a question and then must be able to receive an answer for some random time. It may be the case that he is e.g. asking different services at once and thus he gets answer at a random time, after which he is not willing to get answer from the server any more.

The following IMC is a server accepting ask and after some computation lasting a random time, it provides an answer. Observe that it is not ready to proceed if the other side is no more willing to synchronize on answer.

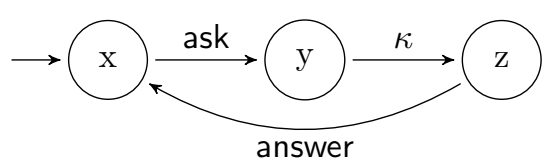

The product is then the following IMC. Observe that after each ask we reach the state $(y, 0)$ with non-zero probability, from where a Now transition leads to a deadlock state $(y, r)$. Since this is an external action, some environments conforming to the specification can ensure that the system eventually ends up almost surely in a deadlock (revealing this error in the implementation of the server).

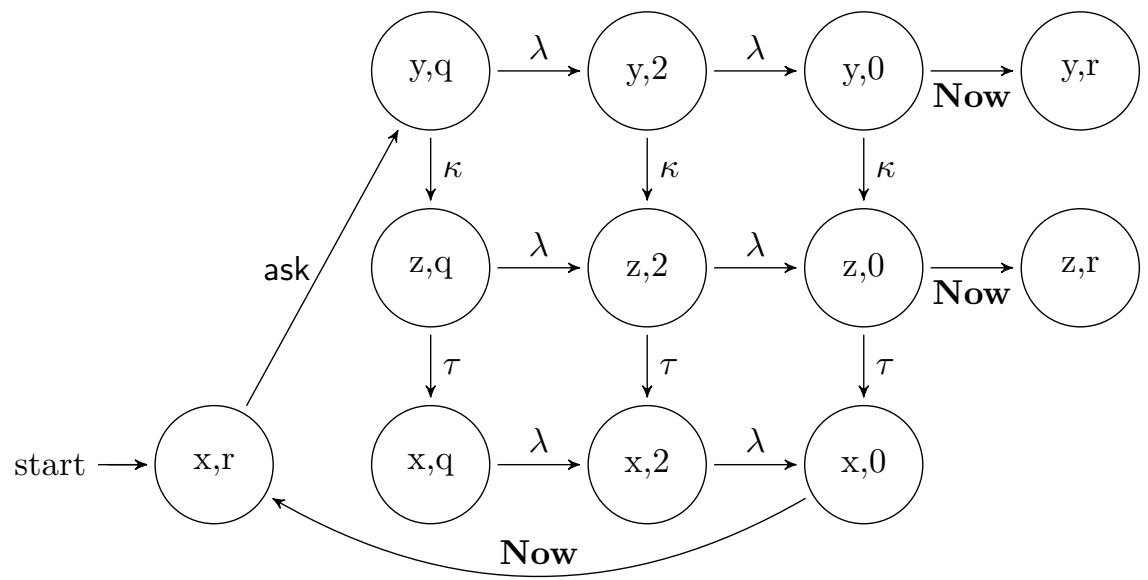




\section{A.2 Product: Hyper-Erlang Phase-Types}

Recall that in $\mathcal{S}_{i}$ each distribution $d$ is replaced with its hyper-Erlang phase-type approximation $d_{i}$ with $i$ branches of lengths 1 to $i$ and rates $\sqrt{i}$ (and rate $2^{2^{i}}$ in the initial state). The only degrees of freedom in the approximation for a fixed $i$ are thus the initial probabilities leading to the branches fo lengths 1 to $i$. For concreteness, we pick a distribution that is lexicographically smallest (w.r.t. the order given by the lengths of branches) such that the resulting cumulative distribution function of time when sink is reached is still pointwise greater than or equal to that of $d$ for the case $\geq d$, and lexicographically largest so that the cdf is pointwise smaller or equal.

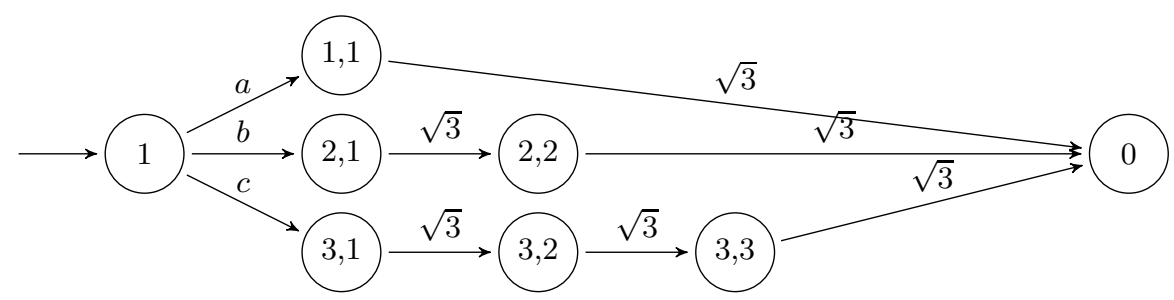

Fig. 1. Hyper-Erlang phase-type for $i=3$ expressing approximately $\frac{a}{256} \cdot \operatorname{Er}(1, \sqrt{3})+$ $\frac{b}{256} \cdot \operatorname{Er}(2, \sqrt{3})+\frac{c}{256} \cdot \operatorname{Er}(3, \sqrt{3})$ with $a+b+c=256$

\section{A.3 Transitions of Commit}

For each commitment $A \subseteq \mathbb{A c t}$ there is an incoming internal transition from commit, an outgoing Change transition to now?, and an outgoing external $a$ transition to commit for each $a \in A$. Furthermore, from now? there is an internal and a Now transition to commit:

- commit $\stackrel{\tau}{\hookrightarrow} A$,

- $A \stackrel{a}{\stackrel{a}{c}}$ commit, for each $a \in A$,

- $A \stackrel{\text { Change }}{\longrightarrow}$ now?,

- now? $\stackrel{\tau}{\hookrightarrow}$ commit and now? $\stackrel{\text { Now }}{\hookrightarrow}$ commit. 


\section{B Proof of Theorem 1}

Theorem 1, For every $\operatorname{IMC} \mathcal{C}$ and $\operatorname{MCA} \mathcal{S}, v_{\mathcal{S}}(\mathcal{C})=\lim _{i \rightarrow \infty} v_{\text {product }}\left(\mathcal{C} \times \mathcal{S}_{i}\right)$, i.e. $\sup _{\sigma \in \mathfrak{S}(\mathcal{C})} \inf _{\substack{\mathcal{E} \in \mathrm{ENV}: \mathcal{E} \models \mathcal{S} \\ \pi \in \mathfrak{S}_{\sigma}(\mathcal{C} \mid \mathcal{E})}} \mathcal{P}_{\mathcal{C} \mid \mathcal{E}}^{\pi}\left[\diamond^{\leq T} G_{\mathcal{E}}\right]=\lim _{i \rightarrow \infty} \sup _{\sigma \in \mathfrak{S}(\mathcal{C})} \inf _{\substack{\mathcal{E} \in \operatorname{ENV} \\ \pi \in \mathfrak{S}_{\sigma}\left(\left(\mathcal{C} \times \mathcal{S}_{i}\right) \mid \mathcal{E}\right)}} \mathcal{P}_{\left(\mathcal{C} \times \mathcal{S}_{i}\right) \mid \mathcal{E}}^{\pi}\left[\diamond^{\leq T} G_{\mathcal{E}}\right]$

\section{Proof idea}

" $\leq$ ": Given an environment $\mathcal{E}$ over $\mathbb{A c t}^{\tau}$ and a scheduler $\pi$, we construct $\mathcal{E}^{\prime}$ satisfying $\mathcal{S}$ that will ensure the same value with the same $\pi$. Intuitively, $\mathcal{E}^{\prime}$ is a composition of $\mathcal{E}$ and $\mathcal{S}$ such that when composed with $\mathcal{C}$, we obtain $(\mathcal{C} \times \mathcal{S}) \mid \mathcal{E}$. Formally, this is exactly $\mathcal{E}^{\prime}:=\mathcal{E} \|_{\mathbb{A} c t} \overline{\mathcal{S}}$ where all "barred" transitions are renamed to unbarred afterwards.

" $\geq$ ": Given an environment $\mathcal{E}$ satisfying $\mathcal{S}$, we construct a sequence of environments $\mathcal{E}_{i}$ to be composed with $\mathcal{C} \times \mathcal{S}_{i}$ that monitor the changes of $\mathcal{S}$ and behave in such a way that together with $\mathcal{C} \times \mathcal{S}$ simulate the original $\mathcal{E}$. The hyper-Erlang form of the phase-type allows for arbitrary precise monitoring. Technically, the environment $\mathcal{E}_{i}$ takes with very high frequency transitions to a special new state, where it checks the progress of $\mathcal{S}$ and simulates the corresponding behaviour of $\mathcal{E}$.

\section{Proof}

" $\leq "$ :

We show the inequality holds for any $i$. Given $i \in \mathbb{N}, \sigma \in \mathfrak{S}(\mathcal{C}), \mathcal{E} \in \mathrm{ENV}^{\prime}, \pi \in$ $\mathfrak{S}_{\sigma}\left(\left(\mathcal{C} \times \mathcal{S}_{i}\right) \mid \mathcal{E}\right)$, we construct $\mathcal{E}^{\prime}$ such that

$$
\mathcal{C}\left|\mathcal{E}^{\prime}=\left(\mathcal{C} \times \mathcal{S}_{i}\right)\right| \mathcal{E}
$$

and hence $\pi$ is a scheduler over both systems and yields the same value on both systems.

We construct $\mathcal{E}^{\prime \prime}=\mathcal{S}_{i} \|_{\mathbb{A c t}} \mathcal{E}$ and then $\mathcal{E}^{\prime}$ by renaming $\bar{a}$ actions to $a$, for each $a \in \mathbb{A c t}^{\tau}$. By case distinction, it is easy to see that the very same transitions are created in $\mathcal{C} \mid \mathcal{E}^{\prime}$ and in $\left(\mathcal{C} \times \mathcal{S}_{i}\right) \mid \mathcal{E}$.

Furthermore, we need to prove that $\mathcal{E}^{\prime} \models \mathcal{S}$. We show that

$\left\{((s, e), s) \mid s\right.$ state of $\mathcal{S}_{i}$ corresponding to a state of $\mathcal{S}$ and $e$ state of $\left.\mathcal{E}^{\prime}\right\}$

is a satisfaction relation. Observe that whenever there is a may transition in the specification, there is a corresponding transition in $\mathcal{S}_{i} \|_{\text {Act }} \mathcal{E}$, and if, moreover, there is a must transition, then there is also an $\bar{a}$, which is then renamed to $a$. Further, we need to define the variable Stop when in state $(s, e)$ and with $s \stackrel{\bowtie d}{\rightsquigarrow} s^{\prime}$. Since the state space of $\mathcal{E}^{\prime}$ is a product of state spaces of $\mathcal{S}_{i}$ and $\mathcal{E}$, we can define Stop to return

- time when the first component becomes $s^{\prime}$ on runs that do not leave states of the phase-type corresponding to $s$ meanwhile; 
- an arbitrary time on the remaining runs, which leave the states of the phasetype before reaching a state with $s^{\prime}$ in the first compoment, so that the cumulative distribution function of Stop is $\bowtie d$.

Observe that such a definition is possible because the cumulative distribution function of Stop conditioned by runs of the first item above satisfies $\bowtie d$. For $\geq d$, the transition Now is only available after the phase-type has elapsed, which takes at least $d$. Hence before time (distributed by) $d, s^{\prime}$ can only be reached using a transition of the form $s \stackrel{a}{\rightarrow} s^{\prime \prime}$ for $s \neq s^{\prime \prime}$ complying the definition of the semantics. For $\geq d$, the phase-type elapses in time (distributed by) at most $d$ and the sink is identified with $s^{\prime}$ hence is reached on time (if no non-looping may transition is taken meanwhile) which is again according to Definition 4

" $\geq ":$

For $\sigma \in \mathfrak{S}(\mathcal{C}), \mathcal{E} \models \mathcal{S}, \pi \in \mathfrak{S}_{\sigma}(\mathcal{C} \mid \mathcal{E})$ and for each $\varepsilon>0$, we construct a sequence $\left(\mathcal{E}_{i}, \pi_{i}\right)_{i \in \mathbb{N}}$ such that

$$
\mathcal{P}_{\mathcal{C} \mid \mathcal{E}}^{\pi}\left[\diamond^{\leq T} G_{\mathcal{E}}\right] \geq \lim _{n \rightarrow \infty} \mathcal{P}_{\left(\mathcal{C} \times \mathcal{S}_{i}\right) \mid \mathcal{E}_{i}}^{\pi_{i}}\left[\diamond^{\leq T} G_{\mathcal{S}_{i}, \mathcal{E}}\right]-\varepsilon
$$

Recall that in $S_{i}$ each distribution $d$ is replaced by a hyper-Erlang distribution with $i$ branches (of all lengths up to $i$ with rates $\sqrt{i}$. A branch of length $\ell$ corresponds to a time $\ell / \sqrt{i}$ it takes to walk through it on average, and for great $i$ 's almost precisely by the law of large numbers. The initial branching probabilities correspond to the probability of this time according to the distribution $d$. In the limit, the hyper-Erlang thus corresponds to the pdf of $d$. Indeed, since for $i$ we have branches taking from $1 / \sqrt{i}$ to $\sqrt{i}$, we cover the whole interval $(0, \infty)$ in the limit.

We define $\mathcal{E}_{i}$ by actions $\mathbb{A} c t \cup\{$ Now $\}$, the state space is $2^{\mathbb{A} c t} \cup \mathbb{A} c t \cup\{$ now?, commit $\} \cup$ $\{0,1\} \times\{1, \ldots, i\}$ with commit being the initial state. For each $A \subseteq \mathbb{A c t}$, there are transitions

- commit $\stackrel{\tau}{\hookrightarrow} A$,

- $A \stackrel{a}{\hookrightarrow} a$ and $a \stackrel{\tau}{\hookrightarrow}$ commit, for each $a \in A$,

- $A \stackrel{2^{i}}{\rightsquigarrow}(0,0)$, and $A \stackrel{2^{i}}{\rightsquigarrow}(1,0)$,

- $(b, j) \underset{\sim}{\stackrel{2^{i}}{\rightarrow}}(0, j+1)$, and $(b, j) \stackrel{2^{i}}{2^{2}}(1, j+1)$ for all $j<i$ and $b \in\{0,1\}$,

- $(b, i) \stackrel{2^{i}}{\stackrel{n}{N} \text { now }}$ ? for $b \in\{0,1\}$,

- now? $\stackrel{\text { Now }}{\longrightarrow}$ commit and now? $\stackrel{\tau}{\rightarrow}$ commit.

In the state commit, $\pi$ can perform any sequence of external transitions changing its commitment after each of them (see Section 5); or a Markovian transition occurs after which a sequence of $i$ random bits is generated; afterwards, $\pi$ returns back to commit (possibly synchronizing over Now).

Intuitively, the scheduler $\pi_{i} \in \mathfrak{S}_{\sigma}\left(\left(\mathcal{C} \times \mathcal{S}_{i}\right) \mid \mathcal{E}_{i}\right)$ simulates behaviour of $\mathcal{C} \mid \mathcal{E}$ in such a way that it is never limited by $\mathcal{S}_{i}$ (as $\mathcal{E}$ anyway satisfies $\mathcal{S}$, there is no reason for further limitations). The transitions under $2^{i}$ create a sequence of random numbers we remember in the current path. These numbers help to 
identify, which set of runs is $\pi$ now simulating. At all times, the current path of $\left(\mathcal{C} \times \mathcal{S}_{i}\right) \mid \mathcal{E}_{i}$ induces a set of paths of $\mathcal{C} \mid \mathcal{E}$.

Each run of $\left(\mathcal{C} \times \mathcal{S}_{i}\right) \mid \mathcal{E}_{i}$ is divided into phases. A phase starts when the current state $(c, s, e)$ is changed to $\left(c^{\prime}, s^{\prime}, e^{\prime}\right)$ with $s \neq s^{\prime}$, i.e. the specification enters another state. We show how a path $\mathfrak{p}$ of length $k$ that starts at the beginning of a new phase induces a set of paths $X_{k}$ in $\mathcal{C} \mid \mathcal{E}$.

With probability $\rightarrow 1$ for $i \rightarrow \infty$, the first next step is the Markovian transition of rate $2^{2^{i}}$ in $\mathcal{S}_{i}$ leading into one of the Erlang branches and thus determining very precisely (for great $i$ 's) how long the phase is going to take. Assume it is of length $\ell$, thus taking time close to $t=\ell / \sqrt{i}$. Further, the choice of the $i$-th branch corresponds to the interval $[x, y)$ (on the $y$-axes) in the cumulative distribution function of the hyper-Erlang phase-type distribution with $x=\sum_{j=0}^{i-1} a_{j}$ and $y=\sum_{j=0}^{i} a_{j}$ where $a_{j}$ is the probability of taking the $j$-th branch.

Since $\mathcal{E}=\mathcal{S}$, there is a random variable $S t o p$ on the runs from $(c, e)$ in $\mathcal{C} \mid \mathcal{E}$ satisfying the condition of the definition of MCA semantics. The interval $[x, y)$ on the $y$-axes in the CDF of Stop corresponds to a interval $[u, v)$ of times on the $x$-axes. As the CDF of Stop is pointwise $\bowtie$ the CDF of the hyper-Erlang, for large enough $i$ the expected value $t$ of time to wait in $\mathcal{S}_{i}$ lies outside of the interval $[u, v)$. Hence, when simulating the set of runs $X_{1}=\{\rho \mid \operatorname{Stop}(\rho) \in[u, v)\}$, the scheduler $\pi_{i}$ is with probability $\rightarrow 1$ for $i \rightarrow \infty$ not limited by $\mathcal{S}_{i}$.

Part of the behavior of runs $X_{1}$ is determined by the randomness in $\mathcal{C}$ and $\sigma$, rest of the behavior is determined by the randomness in $\mathcal{E}$ and $\pi$. After each step $k$ of the phase, the simulating strategy $\pi_{i}$ figures out the currently valid subset $X_{k} \subseteq X$ that conform with the path $\mathfrak{p}$ in the phase so far.

- If in the $k$-th step a Markovian or internal transition is taken in $\mathcal{C}, X_{k}$ is defined as those runs of $X_{k-1}$ with the very same move at the very same time (relative to the start of the phase).

- If in the $k$-th step a Markovian transition within the hyper-Erlang of $\mathcal{S}_{i}$ is taken, this move is ignored by $X_{k}=X_{k-1}$.

- If in the $k$-th step the Markovian transition to now? in $\mathcal{E}_{i}$ is taken, we call this moment a emphcontrol point. The strategy $\pi_{i}$ has a fresh sequence of $i$ random bits. The strategy $\pi_{i}$ divides the runs of $X_{k-1}$ into $2^{i}$ sets of equal measure (conditioned by $X_{k-1}$ ) according to the sequence of synchronization performed in these runs since the last control point and according to the current state. This yields with probability $\rightarrow 1$ for $i \rightarrow \infty$ only constantly many types of this discrete behavior, hence the number of sets with more than one type of behavior remains constant. Using the random sequence, one such set is assigned to $X_{k}$. The strategy simulates the type of discrete behavior with most measure (conditioned by $X_{k}$ ): it performs the sequence of synchronization and moves into a commit according to the current state in $X_{k}$.

After a phase is finished, i.e. when $\mathcal{S}_{i}$ takes the $\ell$ th exponential transition, the transition Now is taken and a new phase begins. The overall induced path is just the concatenation of the paths induced by the previous phases and the current one. 
Now we discuss the possible reasons why the behavior in the simulating IMC might differ from the behavior in $X_{k}$ w.r.t. time-bounded reachability.

- If at any point an Erlang branch of a specification finishes in time outside the assumed interval, $\pi_{i}$ further behaves arbitrarily. However, the measure of these runs tends $\rightarrow 0$ for $i \rightarrow \infty$ due to the weak law of large numbers.

- Each synchronization occuring at time $w$ between two control points is simulated later - at the closest control point at time $w^{\prime}>w$. The behavior of the simulating system may be different from the simulated system if

- a Markovian transition in $\mathcal{C}$ occurs in the interval $\left[w, w^{\prime}\right]$. The number of synchronizations that can occur up to time $T$ is bounded by a constant multiple of the number of Markovian transitions that are taken in $\mathcal{C}$, due to the acyclicity assumption. The sum of lengths of such intervals $\left[w, w^{\prime}\right]$ where a Markovian transition causes trouble thus tends to 0 with probability $\rightarrow 1$ for $i \rightarrow \infty$. Hence, this is not a problem.

- The scheduler takes different decisions because the synchronization has been delayed to $w^{\prime}$. Notice that this occurs only if $w<n \delta<w^{\prime}$ for some $n \in \mathbb{N}$ (due to our assumption on the set of strategies of con). As the points $w$ where synchronization occurs are randomly generated by exponential transitions of $\mathcal{C}$ or $\mathcal{E}$ and the length of the interval $\left[w, w^{\prime}\right]$ tends to 0 , the probability of this behavior also tends to 0 as $i \rightarrow \infty$. 


\section{Proof of Theorem 2}

Theorem 2, For every $\operatorname{IMC} \mathcal{C}, \operatorname{MCA} \mathcal{S}, i \in \mathbb{N}$, we have $v_{\mathcal{G}_{i}}=v_{\text {product }}\left(\mathcal{C} \times \mathcal{S}_{i}\right)$, i.e.

$$
\sup _{\sigma \in \Sigma} \inf _{\pi \in \Pi} \mathcal{P}_{\mathcal{G}_{i}}^{\sigma, \pi}\left[\diamond^{\leq T} G\right]=\sup _{\sigma \in \mathfrak{S}(\mathcal{C})} \inf _{\substack{\mathcal{E} \in \mathrm{ENV} \\ \pi \in \mathfrak{S}_{\sigma}\left(\left(\mathcal{C} \times \mathcal{S}_{i}\right) \mid \mathcal{E}\right)}} \mathcal{P}_{\left(\mathcal{C} \times \mathcal{S}_{i}\right) \mid \mathcal{E}}^{\pi}\left[\nabla^{\leq T} G_{\mathcal{E}}\right]
$$

\section{Proof idea}

" $\leq$ ": We can simulate every $\mathcal{E}$ and scheduler $\pi$ by a strategy of env. The random waiting of $\mathcal{E}$ determined by occurrence of Markovian transitions can be simulated by env by choosing the delays randomly according to the exponential distribution with the respective rate. Further, in each state of $\mathcal{E}$ only some external actions are available and env simulates this by changing the commitment to exactly this set of actions.

" $\geq$ ": Every strategy of env can be (approximately) implemented using a suitable environment $\mathcal{E}$ together with a scheduler $\pi$. We need to simulate the discrete delays chosen by env using random delays available in $\mathcal{E}$. A delay $t$ is simulated by many repetitions of a special Markovian transition with a very fast rate $\lambda$. After the total time adds up to at least $t, \pi$ stops repeating it and continues simulating the discrete transitions of env. We get the result by taking $\lambda \rightarrow \infty$.

\section{Proof}

$$
\text { " } \leq " \text { : }
$$

This amounts to showing that an arbitrary environment $\mathcal{E}$ can be "simulated" by the player env in the CE game. Formally, it is sufficient to prove

$$
\begin{aligned}
\forall \sigma \in \Sigma \exists \sigma^{\prime} \in \mathfrak{S}(\mathcal{C}) \forall \mathcal{E} \in \mathrm{ENV}^{\prime} \forall \pi \in & \mathfrak{S}_{\sigma^{\prime}}\left(\left(\mathcal{C} \times \mathcal{S}_{i}\right) \mid \mathcal{E}\right) \exists \pi_{\mathcal{E}} \in \Pi: \\
& \mathcal{P}_{\mathcal{G}_{i}}^{\sigma, \pi_{\mathcal{E}}}\left[\diamond^{\leq T} G\right] \leq \mathcal{P}_{\left(\mathcal{C} \times \mathcal{S}_{i}\right) \mid \mathcal{E}}^{\pi}\left[\diamond^{\leq T} G\right]
\end{aligned}
$$

Note that every strategy $\sigma$ of the player con is actually also a scheduler for $\mathcal{C}$ (and vice versa). Thus we set $\sigma^{\prime}:=\sigma$ and then for every environment $\mathcal{E}$ and its scheduler $\pi$, we give a strategy $\pi_{\mathcal{E}}$ of the player env that makes "equivalent" decisions as $\pi$ in the "equivalent" path. We then prove that $\pi_{\mathcal{E}}$ guarantees the same value as $\pi$ of $\mathcal{E}$ does.

The idea of the simulation is the following. Whenever $\pi$ synchronizes on an external action $a, \pi_{\mathcal{E}}$ chooses $a$. Whenever $\mathcal{E}$ waits with a rate $\lambda, \pi_{\mathcal{E}}$ chooses to wait, too. Here we use randomizing strategies so that we can combine all waiting times $t \in \mathbb{R}_{>0}$ with the exponential distribution with rate $\lambda$. In other words, $\pi_{\mathcal{E}}$ simulates the random waiting of $\mathcal{E}$ using randomizing.

Let thus $\sigma, \mathcal{E}, \pi$ be arbitrary but fixed. In the following, we define $\pi_{\mathcal{E}}$ through a function WC : Histories $\left(\mathcal{G}_{i}\right) \rightarrow \operatorname{Paths}\left(\left(\mathcal{C} \times \mathcal{S}_{i}\right) \mid \mathcal{E}\right)$ transforming the paths of the $\mathrm{CE}$ game into paths of $\left(\mathcal{C} \times \mathcal{S}_{i}\right) \mid \mathcal{E}$, which $\pi_{\mathcal{E}}$ uses to ask what $\pi$ would do. 
Since $\mathcal{E}$ can have probabilistic branching and $\pi$ can be randomizing, we need to pick one of possibly more paths of $\left(\mathcal{C} \times \mathcal{S}_{i}\right) \mid \mathcal{E}$ corresponding to the history of the simulating play in $\mathcal{G}_{i}$. We will pick one where the future chances are the best for the environment, i.e. worst for the time bounded reachability, hence $\mathrm{WC}$ for the "worst case".

The functions $\pi_{\mathcal{E}}$ and WC are defined inductively and only on the reachable histories; one can define them arbitrarily elsewhere. We start with $\mathrm{WC}\left(c_{0}, q_{0}\right.$, commit $):=$ $\left(c_{0}, q_{0}, e_{0}\right)$. For history $\mathfrak{h}$ ending with some $t(c, q, \bar{e})$ (for the initial one-state path $t=0)$ with $\mathrm{WC}(\mathfrak{h})$ ending in $\left(c, q, e_{1}\right)$, we first define what $\pi$ does after a (possibly empty) sequence of internal steps in $\mathcal{E}$. Let $\left(c_{1}, q, e_{1}\right), \ldots,\left(c_{n}, q, e_{n}\right)$ be such maximal sequence with $\pi\left(\mathrm{WC}(\mathfrak{h}) t\left(c, q, e_{2}\right) t \cdots t\left(c, q, e_{i}\right)\right)\left(c, q, e_{i+1}\right)>0$ and $e_{i} \stackrel{\mathcal{\tau}}{\rightarrow} e_{i+1}$ for $i<n$ that minimizes

$$
\mathcal{P}_{\left(\mathcal{C} \times \mathcal{S}_{i}\right) \mid \mathcal{E}}^{\pi}\left[\diamond^{\leq T} G_{\mathcal{E}} \mid \mathrm{WC}(\mathfrak{h}) t\left(c, q, e_{2}\right) t \cdots t\left(c, q, e_{n}\right)\right]
$$

If $n=1$ then $\left(c, q, e_{2}\right) \cdots\left(c, q, e_{n}\right)$ is empty. This way, although $\pi$ is randomizing, we choose a single choice in a unique way which, moreover, is the best one for the environment.

Depending on the type of the last state $(c, q, \bar{e})$ of $\mathfrak{h}$ and the scheduler's decision dist $:=\pi\left(\mathrm{WC}(\mathfrak{h}) t\left(c, q, e_{2}\right) t \cdots t\left(c, q, e_{n}\right)\right)$, we define $\pi_{\mathcal{E}}(\mathfrak{h})$ as follows:

- If $(c, q, \bar{e})$ is an immediate state and $\bar{e}=$ commit, then

$$
\pi_{\mathcal{E}}(\mathfrak{h}):=A \mapsto 1 \text { for } A \text { the set of actions available in } e_{n},
$$

$\mathfrak{h}^{\prime}:=\mathfrak{h} t(c, q, A)$ and we set $\mathrm{WC}\left(\mathfrak{h}^{\prime}\right):=\mathrm{WC}(\mathfrak{h}) t\left(c, e_{2}\right) t \cdots t\left(c, e_{n}\right)$.

- If $(c, q, \bar{e})$ is an immediate state and $\bar{e} \subseteq \mathbb{A} c t$, then

since $\pi$ respects $\sigma$, there is $p \in[0,1]$ such that dist $=p \cdot \sigma\left(\mathrm{WC}(\mathfrak{h})_{\mathcal{C}}\right)+(1-$ $p) \cdot$ dist $^{\prime}$, so we set

$$
\pi_{\mathcal{E}}(\mathfrak{h}):=(\checkmark \mapsto p)+(1-p) \cdot \text { dist }^{\prime}
$$

Now the next state ( $\tau$-successor) is chosen randomly. The corresponding $\tau$ transition is either

1. a $\tau$ transition of $\mathcal{C}$, or

2. a result of composing $a$ of $\mathcal{C}$ and $\bar{a}$ of $\mathcal{S}_{i}$, or

3. a result of composing $a$ of $\mathcal{C}$ and $a$ of $\mathcal{S}_{i}$ and $a$ of Commit,

we thus obtain the next state $\left(c^{\prime}, q^{\prime}, \bar{e}^{\prime}\right)$. and a new history $\mathfrak{h}^{\prime}:=\mathfrak{h} t\left(c^{\prime}, q^{\prime}, \bar{e}^{\prime}\right)$ and we set $\mathrm{WC}\left(\mathfrak{h}^{\prime}\right):=\mathrm{WC}(\mathfrak{h}) t\left(c, q, e_{2}\right) t \cdots t\left(c, q, e_{n}\right)\left(c^{\prime}, q^{\prime}, e^{\prime}\right)$ where $e^{\prime}=e_{n}$ in the first two cases and $e_{n} \stackrel{a}{\hookrightarrow} e^{\prime}$ in the third case.

4. a result of composing Now of $\mathcal{S}_{i}$ and Now of Commit: we cannot simulate this in $\bar{e} \subseteq \mathbb{A}$ ct, but since $\mathcal{E} \in \mathrm{ENV}^{\prime}$, this case can only happen right after a Markovian transition of $\mathcal{E}$ or $\mathcal{S}_{i}$. Therefore, we first discuss the corresponding timed states and we deal with this case below.

- If $(c, q, \bar{e})$ is a timed state and $\bar{e} \subseteq \mathbb{A} c t$, then only Markovian transition(s) are enabled and dist is ignored. 
- If there are no Markovian transitions available in $e_{n}, / * \mathcal{E}$ is blocked $* /$ we set

$$
\pi_{\mathcal{E}}(\mathfrak{h}):=(T+1) \mapsto 1
$$

the new history is then either longer than $T$ if no Markovian transition from $c$ or $q$ occurs before $T$, or else a Markovian transition occurs after $m$ still before $T$ and we set $\mathfrak{h}^{\prime}=\mathfrak{h}(t+m)\left(c^{\prime}, q^{\prime}, e_{n}\right)$ given by the respective Markovian successor and further $\mathrm{WC}\left(\mathfrak{h}^{\prime}\right):=\mathrm{WC}(\mathfrak{h}) t\left(c, q, e_{2}\right) t \cdots t\left(c, q, e_{n}\right)(t+$ $m)\left(c^{\prime}, q^{\prime}, e_{n}\right)$.

- Else we set

$$
\pi_{\mathcal{E}}(\mathfrak{h}):=\operatorname{Exp}\left(\mathrm{R}\left(e_{n}\right)\right)
$$

from which the respective delay $d$ is sampled. Then either a Markovian transition of $\mathcal{C}$ or $\mathcal{S}$ happens before $d$, in which case $\mathfrak{h}^{\prime}$ and $\mathrm{WC}\left(\mathfrak{h}^{\prime}\right)$ are defined as in the previous case; or else pick arbitrary $e^{\prime}$ with $e_{n} \rightsquigarrow e^{\prime}$ minimizing

$$
\mathcal{P}_{\left(\mathcal{C} \times \mathcal{S}_{i}\right) \mid \mathcal{E}}^{\pi}\left[\diamond^{\leq T} G \mid \mathrm{WC}\left(\mathfrak{h}^{\prime}\right)\right]
$$

where $\mathfrak{h}^{\prime}:=\mathfrak{h}(t+d)(c, q$, now? $)$ and $\mathrm{WC}\left(\mathfrak{h}^{\prime}\right):=\mathrm{WC}(\mathfrak{h}) t\left(c, q, e_{2}\right) t \cdots t\left(c, q, e_{n}\right)(t+$ d) $\left(c, q, e^{\prime}\right)$.

We distinguish three cases of what happens after a timed transition.

- If a Markovian transition of $\mathcal{C}$ wins, we proceed in the standard way.

- If the delay of env wins then $e^{\prime}=$ now? and the state is thus immediate. Let $p$ be the probability that $\pi\left(\mathrm{WC}\left(\mathfrak{h}^{\prime}\right)\right)$ chooses a transition stemming from Now $\left(q \stackrel{\text { Now }}{\hookrightarrow} q^{\prime \prime}, e^{\prime} \stackrel{\text { Now }}{\hookrightarrow} e^{\prime \prime}\right)$. We let $\pi_{\mathcal{E}}$ choose Now also with $p$, and $\tau$ to commit with $1-p$.

In the former case, the new history is $\mathfrak{h}^{\prime \prime}:=\mathfrak{h}^{\prime}(t+d)\left(c, q^{\prime \prime}\right.$, commit $)$ and $\mathrm{WC}\left(\mathfrak{h}^{\prime \prime}\right)=\mathrm{WC}\left(\mathfrak{h}^{\prime}\right)(t+d)\left(c, q^{\prime \prime}, e^{\prime \prime}\right)$.

In the latter case, the new history is $\mathfrak{h}^{\prime \prime}:=\mathfrak{h}^{\prime}(t+d)(c, q$, commit $)$ and $\mathrm{WC}\left(\mathfrak{h}^{\prime \prime}\right)=\mathrm{WC}\left(\mathfrak{h}^{\prime}\right)$.

- If a Markovian transition of $\mathcal{S}$ wins we get to $\left(c^{\prime}, q^{\prime}, e_{n}\right)$ in both cases with $e_{n} \subseteq$ Act. This state is either without available $\tau$ from Now, in which case we proceed in the standard way, or with available $\tau$ from Now. The latter happens due to reaching sink in the case of $\geq d$ constraint. Indeed, this is the only case, where Now turns from unavailable to available, and note that the preceding state was timed and $\mathcal{E}$ did not change its state, hence Now indeed was not available.

Let now $p$ be again the probability that $\pi\left(\mathrm{WC}\left(\mathfrak{h}^{\prime}\right)\right)$ chooses a transition stemming from Now $\left(q \stackrel{\text { Now }}{\hookrightarrow} q^{\prime \prime}, e_{n} \stackrel{\text { Now }}{\hookrightarrow} e^{\prime \prime}\right)$. We let $\pi_{\mathcal{E}}$ wait with delay $\delta$ with $p$ (and behave in the standard way with the remaining probability, which is possible as $q$ and $q^{\prime}$ have the same actions available, see the previous paragraph). If we win, we perform the Now and the new history is $\mathfrak{h}^{\prime \prime}:=\mathfrak{h}^{\prime}(t+d)\left(c, q^{\prime}, \bar{e}\right)(t+d+\delta)\left(c, q^{\prime}\right.$, now? $)(t+d+\delta)\left(c, q^{\prime \prime}\right.$, commit $)$ and $\operatorname{WC}\left(\mathfrak{h}^{\prime \prime}\right)=\mathrm{WC}(\mathfrak{h})(t+d+\delta)\left(c, q^{\prime}, e_{n}\right)(t+d+\delta)\left(c, q^{\prime \prime}, e^{\prime \prime}\right)$. Thus, we pretend that the Markovian transition of $\mathcal{S}_{i}$ took by $\delta$ longer and the $\tau$ from Now was executed immediately. If $\delta$ does not win, we define the 
behaviour of the environment arbitrarily. Apparently, for $\delta$ much smaller than inverse of any rate and approaching 0 , the probability that $\delta$ wins is high and the difference in the time distribution of spec approaches 0 . Therefore, it is sufficient to pick $\delta:=1 / 2^{2^{2^{i}}}$ as the fastest rate is $2^{2^{i}}$ (the initial rate of the hyper-Erlang).

Lemma 1. For every $\sigma \in \mathfrak{S}(\mathcal{C}), \mathcal{E} \in \mathrm{ENV}^{\prime}, \pi \in \mathfrak{S}_{\sigma}\left(\left(\mathcal{C} \times \mathcal{S}_{i}\right) \mid \mathcal{E}\right)$, we have

$$
\mathcal{P}_{\left(\mathcal{C} \times \mathcal{S}_{i}\right) \mid \mathcal{E}}^{\pi}\left[\diamond^{\leq T} G\right] \geq \mathcal{P}_{\mathcal{G}_{i}}^{\sigma, \pi \mathcal{E}}\left[\diamond^{\leq T} G\right]
$$

Proof (Idea). If there are no probabilistic choices in $\mathcal{E}$ and $\pi$ is deterministic then the values are the same. Indeed, the only difference of the simulating probabilistic space to the original one is that whenever there is a probabilistic choice, the environment is always "lucky". Since the minimum of elements is never greater than their affine combination, the result follows.

Proof. Formally, we proceed as follows.

Firstly, we define a measure $\mathcal{P}_{\mathcal{G}}^{\mathcal{E}, \pi}$ on infinite histories of $\mathcal{G}$ directly induced by $\mathcal{E}$ and $\pi$. As opposed to $\pi_{\mathcal{E}}$, the probabilistic choices of the environment are reflected here. Let RealStep : $\operatorname{Paths}\left(\left(\mathcal{C} \times \mathcal{S}_{i}\right) \mid \mathcal{E}\right) \rightarrow \mathbb{H}$ istories $\left(\mathcal{G}_{i}\right)$ project all internal transitions of the environment out, i.e. it maps a run $\left(c_{0}, q_{0}, e_{0}\right) t_{0}\left(c_{1}, q_{1}, e_{1}\right) t_{1} \ldots$ to a run $c_{0} t_{0} \cdots$ where each $c_{i} t_{i}$ is omitted whenever $c_{i}=c_{i-1}$ and $e_{i-1} \stackrel{\tau}{\hookrightarrow} e_{i}$. Then we define $\mathcal{P}_{\mathcal{G}_{i}}^{\mathcal{E}, \pi}:=\mathcal{P}_{\left(\mathcal{C} \times \mathcal{S}_{i}\right) \mid \mathcal{E}} \circ$ RealStep $^{-1}$. Clearly, as $\tau$ transitions take no time we have(4)

$$
\mathcal{P}_{\left(\mathcal{C} \times \mathcal{S}_{i}\right) \mid \mathcal{E}}^{\pi}\left[\diamond^{\leq T} G\right]=\mathcal{P}_{\mathcal{G}_{i}}^{\mathcal{E}, \pi}\left[\diamond^{\leq T} G\right]
$$

Secondly, for $j \in \mathbb{N}_{0}$, consider the set $\mathbb{H i s t o r i e s} j \subseteq \mathbb{H}$ istories $\left(\mathcal{G}_{i}\right)$ of histories of length $j$, i.e. after the $j$ th step is taken. Let $\mathbf{p}_{\mathbf{j}} \in \mathcal{D}\left(\mathbb{H}\right.$ istories $\left.{ }_{j}\right)$ denote the transient probability measure according to $\mathcal{P}_{\mathcal{G}_{i}, \pi}^{\mathcal{E}}$ after $j$ steps. Further let $\mathbf{r}_{\mathbf{j}}$ : Histories $_{j} \rightarrow[0,1]$ be given by $\mathbf{r}_{j}(\mathfrak{h})=\mathcal{P}_{\mathcal{G}_{i}}^{\mathcal{E}, \pi}\left[\diamond^{\leq T} G \mid \mathfrak{h}\right]$. Clearly, as states of $G$ are absorbing we have

$$
\mathcal{P}_{\mathcal{G}_{i}}^{\mathcal{E}, \pi}\left[\nabla^{\leq T} G\right]=\int \mathbf{r}_{\mathbf{j}} d \mathbf{p}_{\mathbf{j}}
$$

Thirdly, let $\mathbf{q}_{\mathbf{j}} \in \mathcal{D}$ (Histories $\left.{ }_{j}\right)$ be the transient probability measure according to $\mathcal{P}_{\mathcal{G}_{i}}^{\sigma, \pi_{\mathcal{E}}}$ after the $i$ th step is taken. A simple induction with case distinction from the definition of $\pi_{\mathcal{E}}$ reveals that

$$
\int \mathbf{r}_{\mathbf{j}} d \mathbf{p}_{\mathbf{j}} \geq \int \mathbf{r}_{\mathbf{j}} d \mathbf{q}_{\mathbf{j}}
$$

Indeed, all but two cases preserve equality. The interesting cases are the Markovian transition in $\mathcal{E}$ and the randomized choice of $\pi$. As the minimum of elements is never greater than their affine combination, we obtain the desired inequality.

$\overline{(4) \text { Note that } \mathcal{E}}$ and $\pi[\sigma]$ do not induce any strategy that would copy the IMC behavior completely. For this, one would need the notion of a strategy with a stochastic update, i.e. a strategy that can change its "state" randomly and thus model where in $\mathcal{E}$ the original path currently is. 
Finally, it remains to prove that

$$
\lim _{j \rightarrow \infty} \int \mathbf{r}_{\mathbf{j}} d \mathbf{q}_{\mathbf{j}}=\mathcal{P}_{\mathcal{G}_{i}}^{\sigma, \pi_{\mathcal{E}}}\left[\diamond^{\leq T} G\right]
$$

i.e. that the gains of the gradual replacements of the strategy converge to the gain of the limiting strategy. This follows from $\mathbf{r}_{\mathbf{j}}(\mathfrak{h})$ being zero or one for each path $\mathfrak{h}$ longer than $T$ only depending on the state at time $T$, and from the fact that the set of runs that never exceed $T$ is of zero measure due to the acyclicity assumption.

The previous lemma proves $(\nabla)$ by which the proof of $v_{\mathcal{G}_{i}} \leq v_{\text {product }}\left(\mathcal{C} \times \mathcal{S}_{i}\right)$ is concluded.

$" \geq$

We can divide the proof in two steps:

1. we show that exponential strategies are sufficient for the player env ;

2. any exponential strategy of the player env can be simulated by a specific environment and its scheduler.

For $\lambda \in \mathbb{R}$ we say that a strategy $\pi$ of the player env is exponential with rate $\lambda$ if it chooses to wait solely with the exponential distribution with rate $\lambda$.

The set of all $\lambda$-exponential strategies is denoted by $\Pi_{\lambda}$.

Exponential strategies for the player env are sufficient:

Lemma 2. For any strategy $\sigma$ we have

$$
\inf _{\pi \in \Pi} \mathcal{P}_{\mathcal{G}_{i}}^{\sigma, \pi}\left[\nabla^{\leq T} G\right]=\inf _{\substack{\lambda \in \mathbb{R}_{>0} \\ \pi_{\lambda} \in \Pi_{\lambda}}} \mathcal{P}_{\mathcal{G}_{i}}^{\sigma, \pi_{\lambda}}\left[\diamond^{\leq T} G\right]
$$

Proof (Idea). Intuitively, if $\pi$ chooses to wait for time $t$ and then makes action $a$, the simulating strategy $\pi_{\lambda}$ repeatedly waits for random time with exponential distribution until the sum of the random waiting times exceeds $t$ and then makes action $a$; the larger the rate $\lambda$, the more precise is this simulation.

Proof. First, we restrict the strategies of $\Pi$ so that on $\mathbb{R}_{>0}$ they only pick Dirac distributions, denoted $\Pi^{\prime}$ :

Claim. $\inf _{\pi \in \Pi^{\prime}} \mathcal{P}_{\mathcal{G}_{i}}^{\sigma, \pi}\left[\diamond^{\leq T} G\right]=\inf _{\pi \in \Pi} \mathcal{P}_{\mathcal{G}_{i}}^{\sigma, \pi}\left[\diamond^{\leq T} G\right]$

Proof. We need to simulate $\pi \in \Pi$ by a strategy $\pi^{\prime} \in \Pi^{\prime}$. For a history $\mathfrak{h}$ and $r \in \mathbb{R}_{>0}$, let $v(r)$ be the conditional lower value of the game if $r$ is chosen in $\mathfrak{h}$. The value in $\mathfrak{h}$ is thus $v(\mathfrak{h}):=\int v(r) d \pi(\mathfrak{h})$. By additivity of measure, there is $r$ for which $v(r) \geq v(\mathfrak{h})$. Therefore, picking $r$ instead of $\pi(\mathfrak{h})$ does not decrease the value. 
We fix arbitrary strategies $\sigma \in \Sigma$ and $\pi \in \Pi^{\prime}$. We need to find a sequence of strategies $\pi_{\lambda}$ for any $\lambda$ such that

$$
\mathcal{P}_{\mathcal{G}_{i}}^{\sigma, \pi}\left[\diamond^{\leq T} G\right] \geq \lim _{\lambda \rightarrow \infty} \mathcal{P}_{\mathcal{G}_{i}}^{\sigma, \pi_{\lambda}}\left[\diamond^{\leq T} G\right]
$$

For any $\lambda>0$, we define $\pi_{\lambda}(\mathfrak{h})$ for $\mathfrak{h}=s_{0} t_{0} \cdots t_{n-1} s_{n}$ using $\pi$ as follows. Intuitively, if $\pi$ chooses to wait for time $t$ and then makes action $a$, the simulating strategy $\pi_{\lambda}$ repeatedly waits for random time with exponential distribution until the sum of the random waiting times exceeds $t$ and then makes action $a$; the larger the rate $\lambda$, the more precise is this simulation. Notice that the history of the play with strategy $\pi_{\lambda}$ contains a lot of waiting steps that are not in the history of the play with strategy $\pi$. Therefore, we need a mapping destutter that removes these superfluous waiting steps and replaces them with the single waiting $\pi$ would perform. We define it inductively by destutter $\left(s_{0}\right)=s_{0}$ and for

$$
\mathfrak{h}=\mathfrak{h}^{\prime} s^{\prime} t_{0} s t_{1} s t_{2} \cdots s t_{n} s^{\prime \prime}
$$

where $s^{\prime} \neq s \neq s^{\prime \prime}$ (corresponding to waiting steps of $\pi_{\lambda}$ where the state is not changed, assuming no Markovian self-loops in $\mathcal{C} \times \mathcal{S}$ ) we set

$$
\text { destutter }(\mathfrak{h}):=\operatorname{destutter}\left(\mathfrak{h}^{\prime} s^{\prime} t_{0}^{\prime} s\right) t_{1}^{\prime} s \cdots s t_{k}^{\prime} s^{\prime \prime}
$$

where

$-t_{0}^{\prime}=t_{0}$

$-\pi\left(\right.$ destutter $\left.\left(\mathfrak{h}^{\prime} s^{\prime} t_{0}^{\prime} s\right) \cdots t_{\ell}^{\prime} s\right)=t_{\ell+1}^{\prime}-t_{\ell}^{\prime}$ for all $0 \leq \ell<k-1$,

$-t_{k}^{\prime}=t_{k-1}^{\prime}$ if the last transition was immediate, and $t_{k}^{\prime}=t_{n}$ if the last transition was Markovian of $\mathcal{C} \times \mathcal{S}_{i}$

Furthermore, let $a^{\prime}$ be the first action taken by $\pi$ at total time $t^{\prime}$ for history destutter $(\mathfrak{h})$ if no Markovian transition occurs (notice that strategy $\pi$ may decide to wait subsequently for several times before it chooses an action; $a^{\prime}$ is the first action taken by $\pi$ if none of the waiting is interrupted by a Markovian transition). We finally set $\pi_{\lambda}(\mathfrak{h})$ to choose

$$
\pi_{\lambda}(\mathfrak{h} t s)= \begin{cases}\operatorname{Exp}(\lambda) & \text { if } t<t^{\prime} \\ a^{\prime} & \text { if } t \geq t^{\prime}\end{cases}
$$

the exponential distribution with rate $\lambda$ in timed states and $\pi($ destutter $(\mathfrak{h}))$ in immediate states. Notice that the strategy $\pi_{\lambda}$ is by definition $\lambda$-exponential.

We now define a set of runs $X_{\lambda}$ in the game with $\pi_{\lambda}$ where the imprecision in the simulation does not cause any difference with respect to the time bounded reachability. Let $\delta>0$ be the clock resolution of $\sigma$. A run in the CE game with strategies $\sigma, \pi_{\lambda}$ belongs to $X_{\lambda}$ if for all $k \in\{0,1, \ldots, T / \delta\}$ we have that

- no non-self-loop transition occurs at the total time neither in the interval $[k \delta, k \delta+\delta / \sqrt{\lambda}]$ nor in the interval $[(k+1) \delta-\delta / \sqrt{\lambda},(k+1) \delta]$. 
- the first transition after total time $k \delta$ is a self-loop transition and occurs in the interval $[k \delta, k \delta+\delta / \sqrt{\lambda}]$;

The proof of the lemma is concluded by the following claim.

Claim. For $\lambda \rightarrow \infty$ we have

$$
\begin{aligned}
\mathcal{P}_{\mathcal{G}_{i}}^{\sigma, \pi_{\lambda}}\left[X_{\lambda}\right] & \rightarrow 1 \\
\mathcal{P}_{\mathcal{G}_{i}}^{\sigma, \pi_{\lambda}}\left[\diamond^{\leq T} G \mid X_{\lambda}\right] & \rightarrow \mathcal{P}_{\mathcal{G}_{i}}^{\sigma, \pi}\left[\diamond^{\leq T} G\right]
\end{aligned}
$$

Proof. As regards (11), we deal with the conditions on runs in $X_{\lambda}$ one by one. First, notice that the Lebesgue measure of all the forbidden intervals tend to 0 as $\lambda$ goes to infinity; hence, the probability of a Markovian transition occurring in any such interval tends to 0 . Second, we can underestimate the probability of $X_{\lambda}$ by considering only the waiting transitions of $\pi_{\lambda}$ as self-loops. The probability that the waiting transition occurs in each such interval can be bounded by

$$
\left(1-e^{\lambda \cdot \delta / \sqrt{\lambda}}\right)^{T / \delta}=\left(1-e^{\sqrt{\lambda} \delta}\right)^{T / \delta} \rightarrow 1
$$

since $T / \delta$ is constant and $e^{\sqrt{\lambda} \delta} \rightarrow 0$ as $\lambda \rightarrow \infty$.

As regards (2), notice that the delay caused by the exponential simulation does not qualitatively change the behaviour. Namely, under the condition of $X_{\lambda}$,

- any transition made by $\pi$ is simulated by $\pi_{\lambda}$ at most $\delta / \sqrt{\lambda}$ later; the player con cannot interfere meanwhile because the states are either timed or immediate, never both;

- also no Markovian transition occurs meanwhile;

- the decision of the players after the delayed transition are the same as in the original play, since the first player plays the same in each whole interval $[k \delta,(k+1 \delta)$ and the second player is asked what he would do if the $\lambda$ transition was precisely on time.

The change is only quantitative because we limit the Markovian transitions, but this change tends to zero as the probability of the set we condition by goes to one.

An exponential strategy in $\mathcal{G}$ can be simulated by an IMC environment of $\mathcal{C}$ :

Lemma 3. For any scheduler $\sigma$ we have

$$
\inf _{\substack{\lambda \in \mathbb{R}_{>0} \\ \pi^{\prime} \in \Pi_{\lambda}}} \mathcal{P}_{\mathcal{G}_{i}}^{\sigma, \pi^{\prime}}\left[\nabla^{\leq T} G\right] \geq \inf _{\substack{\mathcal{E} \in \mathrm{ENV}^{\prime} \\ \pi \in \mathfrak{S}_{\sigma}\left(\left(\mathcal{C} \times \mathcal{S}_{i}\right) \mid \mathcal{E}\right)}} \mathcal{P}_{\left(\mathcal{C} \times \mathcal{S}_{i}\right) \mid \mathcal{E}}^{\pi}\left[\diamond^{\leq T} G\right]
$$

Proof (Idea). Since only one rate is used, we can build a "universal" environment (w.r.t. this rate), that can freely select on which actions to synchronize and waiting with exactly this rate. 
Proof. We fix an arbitrary scheduler $\sigma$ and use the same strategy $\sigma$ as before (observe that a scheduler has the same type as a strategy of con). Furthermore, we fix an arbitrary $\lambda \in \mathbb{R}_{>0}$ and a $\lambda$-exponential strategy $\pi^{\prime}$. We choose $\mathcal{E}$ to be the environment of $E N V^{\prime}$ depicted below for $\mathbb{A c t}=\{\mathrm{a}\}$. It is very similar to Commit from Section 5. The action alphabet of $\mathcal{E}$ is $\mathbb{A}$ ct $\cup\{$ Now $\}$, the state space is $2^{\mathbb{A} c t} \cup\{$ commit, now? $\}$ and the transitions are for every $A \subseteq \mathbb{A} c t$

- commit $\stackrel{\tau}{\rightarrow} A$,

$-A \stackrel{a}{\hookrightarrow}$ commit, for each $a \in A$,

- $A \stackrel{\lambda}{\hookrightarrow}$ now?,

- now? $\stackrel{\tau}{\hookrightarrow}$ commit and now? $\stackrel{\text { Now }}{\hookrightarrow}$ commit.

Note that the only rate is $\lambda$. This is in some sense universal environement in $\mathrm{ENV}^{\prime}$ for $\mathcal{C} \times \mathcal{S}$. Its power is only limited by $\lambda$ (for $\lambda \rightarrow \infty$ it can simulate any other environment).

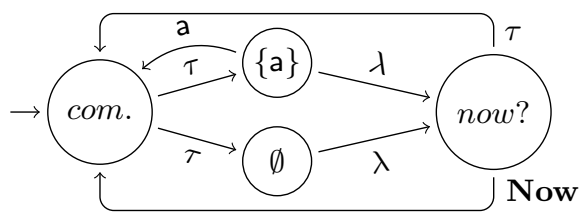

We set $\pi$ to be scheduler that chooses the same transitions as the strategy $\pi^{\prime}$. And when $\pi^{\prime}$ decides to wait exponentially with $\lambda$ in a timed state, we are necessarily in some $A \subseteq \mathbb{A}$ ct and thus automatically wait with $\lambda$ exponential waiting. This definition is correct as the paths of $\left(\mathcal{C} \times \mathcal{S}_{i}\right) \mid \mathcal{E}$ directly correspond to histories of $\mathcal{G}_{i}$.

Since for any measurable set of runs $X$ in $\mathcal{G}_{i}$ we have $\mathcal{P}_{\mathcal{G}_{i}}^{\sigma^{\prime}}{ }^{\prime}[X]=\mathcal{P}_{\left(\mathcal{C} \times \mathcal{S}_{i}\right) \mid \mathcal{E}_{\lambda}}^{\sigma, \pi}\left[\operatorname{proj}_{1}^{-1}(X)\right]$ we also hav

$$
\mathcal{P}_{\mathcal{G}^{\prime}}^{\sigma^{\prime}, \pi^{\prime}}[\diamond \leq T G]=\mathcal{P}_{\left(\mathcal{C} \times \mathcal{S}_{i}\right) \mid \mathcal{E}_{\lambda}}^{\pi}\left[\diamond^{\leq T} G\right]
$$

Finally, the proof of $v_{\mathcal{G}_{i}} \geq v_{\text {product }}\left(\mathcal{C} \times \mathcal{S}_{i}\right)$ follows easily from Lemmata 2, and 3 since we have

$$
\sup _{\sigma \in \Sigma} \inf _{\pi \in \Pi} \mathcal{P}_{\mathcal{G}_{i}}^{\sigma, \pi}\left[\nabla^{\leq T} G\right]=\sup _{\sigma \in \Sigma} \inf _{\substack{\lambda \in \mathbb{R}_{>0} \\ \pi_{\lambda} \in \Pi_{\lambda}}} \mathcal{P}_{\mathcal{G}}^{\sigma, \pi_{\lambda}}\left[\nabla^{\leq T} G\right] \geq \sup _{\sigma \in \Sigma} \inf _{\substack{\mathcal{E} \in \mathrm{ENV}^{\prime} \\ \pi \in \mathfrak{S}_{\sigma}\left(\left(\mathcal{C} \times \mathcal{S}_{i}\right) \mid \mathcal{E}\right)}} \mathcal{P}_{\left(\mathcal{C} \times \mathcal{S}_{i}\right) \mid \mathcal{E}}^{\pi}\left[\nabla^{\leq T} G\right]
$$




\section{C.1 Proof of Theorem 3}

Theorem 3. For every IMC $\mathcal{C}$, MCA $\mathcal{S}$, we have $v_{\mathcal{G}}=\lim _{i \rightarrow \infty} v_{\mathcal{G}_{i}}$.

\section{Proof idea}

The states of the hyper-Erlang phase-type give approximate information about the remaining time in the current state of the specification. Moreover, this time is known shortly after entering the phase-type: in $\mathcal{G}_{i}$ after taking the first (fast) transition, in $\mathcal{G}$ after an arbitrarily short time chosen by env. Furthermore, the greater the hyper-Erlang, the more precise time estimation we have. In the limit, we thus know (from after the first transition till the sink) what the sampled remaining time exactly is. We can thus provide simulations back and forth.

\section{Proof}

" $\leq$ ":

We need to simulate $\pi_{i}$ of $\mathcal{G}_{i}$ for a given fixed $i$. Here it is sufficient to:

- upon entering a specifcation state wait with time distributed according to the first rate $2^{2^{i}}$ of the respective hyper-Erlang, and decide which branch $j$ we take in the simulated $\mathcal{G}_{i}$ (see below) when we get the sampled time $t$ in $\mathcal{G}$

- simulate $k$ th Markovian transition of the hyper-Erlang branch $j$ : here we simply randomly choose time for this Markovian transition and check whether it happens before or after the proposed waiting time and perform the earlier (and possibly finish the waitingh later). The time for the Markovian transition is chosen according to the hyper-Erlang rate $\sqrt{i}$ under the condition that we are in the current branch $j$ at the $k$ th node and we should get to sink in time $t-t^{\prime}$ where $t^{\prime}$ is the time spent in the current state $q$ of $\mathcal{S}$.

- All other choices are the same as $\pi_{i}$ does in the respective (straightforwardly defined) history of $\mathcal{G}_{i}$.

It remains to show how to pick which branch to simulate, i.e. choose $j$. Firstly, there is a distribution on which length to choose under the condition that we should reach sink in precisely $t$, denote its cdf by Branch. We consider an arbitrary fixed mapping Indep $:[0,1] \rightarrow[0,1]$ where the argument is independent of the result. Denoting $F_{\sqrt{i}}$ the cdf of exponential distribution with rate $\sqrt{i}$, we $\operatorname{Branch}^{-1}\left(\operatorname{Indep}\left(F_{\sqrt{i}}(t)\right)\right)$. This way, we use the random waiting (that can be seen in the history) as a random generator for the choice of the branch and thus we keep this choice implicitely in the history of the game.

$$
\text { " } \geq \text { ": }
$$

We simulate the behaviour of $\pi$ in $\mathcal{G}$ who has precise information about the time progress of the specification by a $\pi_{i}$ in $\mathcal{G}_{i}$ who only knows his position in the respective hyper-Erlang, so that for $i \rightarrow \infty$ the error approaches 0 . The main idea is that when we reach in the $\mathcal{S}_{i}$ component a state of the form $(j, 1)$ we 
guess how long we have before $\mathcal{S}$ changes its state and then behave according to what $\pi$ would do with this time.

When in $(q, j, 1)$ the cdf to reach sink is say $E_{j}$ (with the mean $j / \sqrt{i}$ ). Further, let $F_{2^{2^{i}}}$ be the cdf of $\operatorname{Exp}\left(2^{2^{i}}\right)$. For time $t$ (which it took to take the $2^{2^{i}}$ transition) and $(j, 1)$ (the reached target of this transition), we define

$$
\operatorname{Time}_{i}(j, t):=E_{j}^{-1}\left(\operatorname{Indep}\left(F_{2^{2^{i}}}(t)\right)\right)
$$

i.e. we use the random quantile of the transition duration to get the random quantile for the time left in the current specification location.

We now define a mapping GetTimes : $\mathbb{H}$ istories $\left(\mathcal{G}_{i}\right) \rightarrow \mathbb{H}$ istories $(\mathcal{G})$. For a history

$\left(c_{0},\left(q_{\text {prev }}, x, y\right), e_{0}\right) t_{1}\left(c_{1},(q, 1,0), e_{1}\right) t_{2}\left(c_{2},(q, j, 1), e_{2}\right) \cdots t_{n}\left(c_{n},(q, j, z), e_{n}\right) t\left(c,\left(q_{n e x t}, 1,0\right), e\right) \mathfrak{h}$

with $q_{\text {prev }} \neq q \neq q_{\text {next }}$ we have $c_{2}=c_{1}$, denote $t^{\prime}=$ Time $_{i}\left(j, t_{2}-t_{1}\right)$, and define the value of GetTimes as follows:

- remove all transitions corresponding to the moves of the specification while its state is still $q$,

- replace every $(q, j, k)$ (as well as $(q, 1,0))$ by $\left(q, t^{\prime}\right)$,

- decrease all times from $t_{2}$ onwards (now also in $\mathfrak{h}$ !) by $t_{2}-t_{1}$,

- if the sink is reached (say at $t_{k}$ ) then replace $t_{k}$ by $t^{\prime}$,

- we process $\left(c_{n},(q, j, z), e_{n}\right) t\left(c,\left(q_{n e x t}, 1,0\right), e\right) t \mathfrak{h}$ the same way. If the end of $\mathfrak{h}$ ends in the middle of a hyper-Erlang branch, the fourth point does not apply.

This way, we pretend the transition from the initial state of the hyper-Erlang took no time and we guessed the correct time to the sink. Observe that for $i \rightarrow \infty$, both errors approach zero.

Let now $\sigma$ be any scheduler (thus a strategy in both $\mathcal{G}_{i}$ and $\mathcal{G}$ ) and $\pi$ a strategy of env in $\mathcal{G}$. We now define $\pi_{i}$. For a history $\mathfrak{h}$ ending at time $t$, we have a history $\overline{\mathfrak{h}}:=\operatorname{GetTimes}(\mathfrak{h})$ ending at time $\bar{t}$. In immediate states, we set $\pi_{i}(\mathfrak{h}):=\pi(\overline{\mathfrak{h}})$. In timed states, (assuming the $\pi$ is deterministic, see Claim in the proof of the previous theorem) we set

$-\pi_{i}(\mathfrak{h}):=\pi(\overline{\mathfrak{h}})$ if $\bar{t}<t$,

$-\pi_{i}(\mathfrak{h}):=\pi(\overline{\mathfrak{h}})-(\bar{t}-t)$ if $\bar{t}>t$ and the result is positive,

$-\pi_{i}(\mathfrak{h}):=\pi(\overline{\mathfrak{h}}) / 2^{i+k}$ otherwise, where $k$ is the length of the current history. (Intuitively, when a hyper-Erlang branch finishes later than it should have according to the guess, we slow down our waiting so that $\pi$ catches up.)

We now define a sequence of sets $\left(X_{\ell}\right)_{\ell \in \mathbb{N}}$ such that for every $\ell$

$$
\lim _{i \rightarrow \infty} \mathcal{P}_{\mathcal{G}_{i}}^{\sigma, \pi_{i}}\left[X_{\ell}\right]=1
$$

and

$$
\lim _{\ell \rightarrow \infty} \lim _{i \rightarrow \infty} \mathcal{P}_{\mathcal{G}_{i}}^{\sigma, \pi_{i}}\left[\diamond^{\leq T} G \mid X_{\ell}\right] \leq \lim _{\ell \rightarrow \infty} \mathcal{P}_{\mathcal{G}}^{\sigma, \pi}\left[\diamond^{\leq T} G\right]
$$

Recall the clock resolution $\delta$. The set $X_{\ell}$ is defined as the set of runs where: 
- no Markovian transitions occur at times in $[k \delta-\delta / \ell, k \delta+\delta / \ell]$ for any $k \in$ $\{1, \ldots, T / \delta\}$

- the sum of durations of all transitions from the initial states of hyper-Erlangs before time $T$ does not exceed $\delta / \ell^{2}$, and

- for each pair of $t^{\prime}, t_{k}$ from above it holds $\left|t^{\prime}-t_{k}\right|<\delta / \ell$.

The first equation clearly holds by the weak law of large numbers and the fact that hyper-Erlangs approximate any continuous distributions.

The second equation then follows because:

- At all moments the last time of $\mathfrak{h}$ is in the same $\delta$-slot $[k \delta,(k+1 \delta))$ as the last time of GetTimes $(\mathfrak{h})$ since we always keep these two aligned, except when $\mathfrak{h}$ is ahead by $x$ and $\pi$ chooses to wait for less than $x$. But then we slow down our progress (see the third line of the definition of $\pi_{i}$ ). Further, under these conditions in total we wait for less than $\sum_{k=1}^{\infty} \delta / 2^{i+k}=\delta / 2^{i}$, which is smaller than $\delta / \ell$ for sufficiently large $i$. Moreover, for sufficiently large $i$, it is even smaller than $\delta / \ell-\delta / \ell^{2}$. Hence by waiting for $\pi$ to catch up, we cannot be pushed out ofthe same slot as GetTime(h) is in, not even because of the inital transitions in the hyper-Erlangs.

- Therefore, $\sigma$ in $\mathcal{G}_{i}$ plays as in the simulated $\mathcal{G}$ since it makes the same decision throughout each whole $\delta$-slot and for each history $\mathfrak{h}$ ending in time $t$, GetTime $(\mathfrak{h})$ ends in time $t^{\prime}$ which is in the same slot.

- Thus for each $\ell$, we finish in the same slot as $\pi$, hence at the same time and state.

The second equation then concludes also the proof of this direction of the theorem. 


\section{Definition of the CE game $\mathcal{G}$}

The CE game $\mathcal{G}$ is defined on the game arena $G$ obtained from the game arena $G_{1}$. First, we need to alter $G_{1}$ a bit. To simplify the argumentation, we assume that in $\mathcal{G}_{1}$, each immediate state has an internal transition that con can choose. If there is none, we add an internal transition to any goal state that each strategy $\sigma$ has to choose with probability 1 . This does not change the value $v_{\mathcal{G}}$ as the strategy $\pi$ can always reject such choice. The internal transitions that con can choose will be denoted by $v \stackrel{\text { con }}{\hookrightarrow} v^{\prime}$, internal transitions that env can choose will be denoted by $v \stackrel{\text { env }}{\hookrightarrow} v^{\prime}$. For each pair of states $v \rightsquigarrow v^{\prime}$ where the Markovian transition corresponds to the flow of time in the specification component with constraint $\bowtie d$, we remove this Markovian transition, and write $v \stackrel{d}{\rightsquigarrow} v^{\prime}$ instead. For each state $v$ for which there is no $v^{\prime}$ and $d$ such that $v \stackrel{d}{\rightsquigarrow} v^{\prime}$, we write $v \stackrel{d}{\rightsquigarrow} v$ for some distribution $d$ from the specification.

The game arena is $G=\left(G_{1} \times\left(\mathbb{R}_{>0}\right)^{2}\right)$. The first real number in a state is the time to wait in the current state of the specification as sketched in the main body of the text. The second real number is artificial, included for later proofs. We set Histories $(\mathcal{G})=\left(G \times \mathbb{R}_{\geq 0}\right)^{*} \times G$, similarly to the definition of $\mathcal{G}_{i}$. We define a sigma-field $\mathfrak{P}$ over $\mathbb{H i s t o r i e s}(\mathcal{G})$ to be the naturally induced product sigmafield $\mathfrak{P}$ where for each discrete component we use the sigma-field induced by the discrete topology and for each real component we use the Borel sigma-field.

A strategy of player con is a measurable function $\sigma: \mathbb{P a t h s}(\mathcal{G}) \rightarrow \mathcal{D}(G)$ and a strategy of player env is a measurable function $\pi: \operatorname{Paths}(\mathcal{G}) \rightarrow \mathcal{D}(\{\checkmark\} \cup G) \cup$ $\mathcal{D}\left(\mathbb{R}_{>0}\right)$, where $\mathbb{B}$ denotes the Borel sigma-field over $\mathbb{R}_{>0}$. For any history $\mathfrak{h}$, we require that $\sigma(\mathfrak{h})$ and $\pi(\mathfrak{h})$ support only finitely many states - those that can be reached by internal transitions where the real component remains intact.

For a given pair of strategies $\sigma, \pi$ we define the semantics of the CE game as a discrete-time Markov chain over the measurable space $(\mathbb{P a t h s}(\mathcal{G}), \mathfrak{P})$. The transition kernel $P$ of this chain, where $P(\mathfrak{h}, A)$ denotes the probability to move in one step from the history $\mathfrak{h}$ to any history in the set $A$, is defined as follows. Let us fix a history $\mathfrak{h}=\left(v_{0}, r_{0}, u_{0}\right) t_{1}\left(v_{1}, r_{1}, u_{1}\right) t_{2}\left(v_{2}, r_{2}, u_{2}\right) \cdots t_{n}\left(v_{n}, r_{n}, u_{n}\right)$ where each $v_{i} \in G_{1}$ and $r_{i}, u_{i} \in \mathbb{R}_{\geq 0}$. Further, we fix a measurable set $A$ of histories.

- If $v_{n}$ is an immediate state, let $d=\sigma(\mathfrak{h})$ and $e=\pi(\mathfrak{h})$. We have

$$
\begin{aligned}
P(\mathfrak{h}, A)= & \sum_{v \in \operatorname{supp}(e)} e(v) \cdot\left[\mathfrak{h} t_{n}\left(v, r_{n}, u_{n}\right) \in A\right] \\
& +e(\checkmark) \cdot \sum_{v_{i} \stackrel{\text { con }}{\hookrightarrow} v_{n+1}} d\left(v_{n+1}\right) \cdot\left[\mathfrak{h} t_{n}\left(v_{n+1}, r_{n}, u_{n}\right) \in A\right]
\end{aligned}
$$

where [condition] is the indicator function of the condition condition. Observe that the first line above corresponds to player env rejecting the choice of con and choosing his own state $v$, the second line corresponds to accepting the choice. 
- If $v_{n}$ is a first timed state visited after a new specification state is entered, the waiting time for the specification is generated as follows.

$P(\mathfrak{h}, A)=\int_{0}^{\infty} \int_{0}^{\infty} u d(r, u) \cdot\left[\mathfrak{h}\left(t_{n}\right)\left(v_{n}, r, u\right) \in A\right] d r d u$

where $u d$ is the density of the uniform distribution over the area $\{(r, x) \mid$ $\left.r \in \mathbb{R}_{>0}, 0<x<f(r)\right\}$ below the curve of $f$ where $f$ is the density of the distribution $d$.

- For other timed states $v_{n}$, let $F=\pi(\mathfrak{h})$. We set

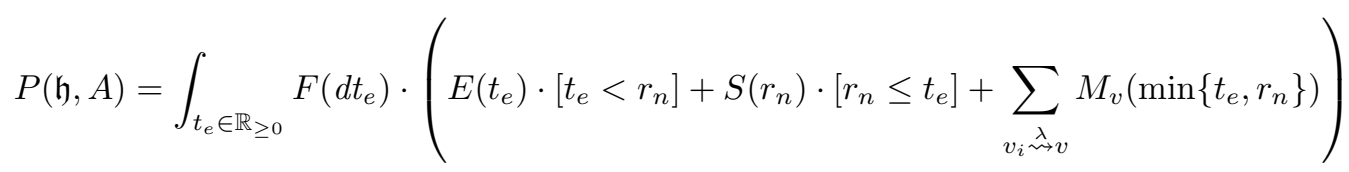

where the terms $E\left(t_{e}\right), S\left(r_{n}\right)$, and $M_{v}(t)$ describe the impact of the Change transition at time $t_{e}$, the flow transition in the specification at time $r_{n}$, and the Markovian transition to $v$ up to time $t$, respectively.

$$
\begin{aligned}
E(t) & =e^{-\mu t} \cdot\left[\mathfrak{h}\left(t_{n}+t\right)\left(v^{\prime}, r_{n}-t, u_{n}\right) \in A\right] \\
S(t) & =e^{-\mu t} \cdot\left[\mathfrak{h}\left(t_{n}+t\right)\left(v^{\prime \prime}, 0, u_{n}\right) \in A\right] \\
M_{v}(t) & =\frac{\lambda}{\mu} \cdot \int_{0}^{t} \mu \cdot e^{-\mu x} \cdot\left[\mathfrak{h}\left(t_{n}+x\right)\left(v, r_{n}-x, u_{n}\right) \in A\right] d x
\end{aligned}
$$

where $v_{n} \stackrel{\lambda}{\rightsquigarrow} v, \mu=\sum_{v_{n} \stackrel{\lambda}{\rightsquigarrow v_{n+1}}} \lambda, v_{n} \stackrel{\text { Change }}{\hookrightarrow} v^{\prime}$, and $v_{n} \stackrel{d}{\rightsquigarrow} v^{\prime \prime}$. 


\section{E Proof of Theorem 4}

Theorem 4, For every IMC $\mathcal{C}$ and MCA $\mathcal{S}, v_{\mathcal{G}}$ is approximated by $v_{\Delta}$ :

$$
\left|v_{\mathcal{G}}-v_{\Delta}\right| \leq 10 \kappa(b T)^{2} \ln \frac{1}{\kappa} .
$$

A strategy $\sigma^{*}$ optimal in $\Delta$ defines a strategy $\left(10 \kappa(b T)^{2} \ln \frac{1}{\kappa}\right)$-optimal in $\mathcal{G}$. Further, $v_{\Delta}$ and $\sigma^{*}$ can be computed in time polynomial in $|\Delta|$, hence in time $2^{\mathcal{O}(|\mathcal{G}|)}$

Proof. The proof is performed in several steps:

1. An approximate game $\mathcal{G}^{\prime}$ is defined where at most one Markovian transition occurs in each interval $[\ell \kappa,(\ell+1) \kappa)$ for $\ell \in \mathbb{N}_{0}$. Furthermore new waiting times for timed transitions in the specification are randomly generated only at times $\ell \kappa$ for $\ell \in \mathbb{N}_{0}$. This game approximates the game $\mathcal{G}$ by the bounds above. No other approximation error is involved in the further steps.

2. A discrete step game $\mathcal{G}^{\prime \prime}$ is defined, which is very similar to $\mathcal{G}^{\prime}$, where every $\kappa$ time units an artificial self-loop is introduced and the set of actions of player env is slightly extended; it is shown to have the same value as $\mathcal{G}^{\prime}$.

3. Thanks to the extended set of actions, a class of grid strategies, which have finite representation, are shown to suffice in $\mathcal{G}^{\prime \prime}$.

4. Thanks to the artificial self-loops in $\mathcal{G}^{\prime \prime}$ and the grid strategies, a discrete stochastic game played on a tree $\Delta$ is obtained directly from $\mathcal{G}^{\prime \prime}$. These games have equal value.

5. The discrete game $\Delta$ is shown to be solved in time polynomial in its (exponential) size.

Formally, these steps are proved in Lemmata 4, 5, 0, and 8

\section{E.1 The approximate game $\mathcal{G}^{\prime}$}

For a fixed $\kappa>0$, we define game $\mathcal{G}^{\prime}$ over the same state space of histories of $\mathcal{G}$ with the same set of strategies. The transition kernel $P^{\prime}$ of $\mathcal{G}^{\prime}$ agrees with $P$ of $\mathcal{G}$ on immediate states, as regards times states there are a few differences. Grid of intervals of length $\kappa$ plays a crucial rule in the semantics.

- In the grid slot where the specification state changes, no Markovian transition occurs. Furthermore, when the specification changes state at time $l \kappa+x$, a new random number is generated as before, but the remaining time till the end of the current interval $\kappa-x$ is added to the newly generated number. Notice that this simulates the situation where the new random number is actually generated at the end of the interval.

- Within one interval of the grid, at most one Markovian transition occurs. 
Formally, let us again fix a history $\mathfrak{h}=\left(v_{0}, r_{0}, u_{0}\right) t_{1} \cdots t_{n}\left(v_{n}, r_{n}, u_{n}\right)$ where each $v_{i} \in G_{1}, r_{i}, u_{i} \in \mathbb{R}_{\geq 0}$, and $v_{n}$ is a timed state. We also fix a measurable set $A$ of histories. Further, let $a$ be the minimal number such that $t_{n}+a=\ell \cdot \kappa$ for some $\ell \in \mathbb{N}$, i.e. the remaining time till a grid line. Let $b$ be the maximal number such that $b<r_{n} b-a=\ell \cdot \kappa$ for some $\ell \in \mathbb{N}$, i.e. the remaining time till the last grid line before the specification changes its state. Finally, we set $c=a$ if there was a Markovian transition in the last $\kappa-a$ time; and we set $c=0$, otherwise. For the first timed state visited after a new specification state is entered, we have

$P^{\prime}(\mathfrak{h}, A)=\int_{0}^{\infty} \int_{0}^{\infty} u d\left(r^{\prime}, u^{\prime}\right) \cdot\left[\mathfrak{h}\left(t_{n}\right)\left(v_{n},\left(r^{\prime}+b+\kappa-r_{n}\right), u^{\prime}\right) \in A\right] d r^{\prime} d u^{\prime}$

whereas for other timed states, we have

$$
\begin{aligned}
P^{\prime}(\mathfrak{h}, A) & =\int_{t_{e} \in \mathbb{R}_{\geq 0}} F\left(d t_{e}\right) \cdot\left(E\left(t_{e}\right) \cdot\left[t_{e}<r_{n}\right]+S\left(r_{n}, b\right) \cdot\left[r_{n} \leq t_{e}\right]+\sum_{\substack{v_{i} \\
\varkappa v}} M_{v}\left(\min \left\{t_{e}, b\right\}\right)\right) \\
E(t) & =e^{-\mu t} \cdot\left[\mathfrak{h}\left(t_{n}+t\right)\left(v^{\prime}, r_{n}-t, u_{n}\right) \in A\right] \\
S(t, b) & \left.=e^{-\mu b} \cdot\left[\mathfrak{h}\left(t_{n}+t\right)\left(v^{\prime \prime}, 0, u_{n}\right)\right) \in A\right] \\
M_{v}(t) & =\frac{\lambda}{\mu} \cdot \int_{c}^{t} \mu \cdot e^{-\mu x} \cdot\left[\mathfrak{h}\left(t_{n}+x\right)\left(v, r_{n}-x, u_{n}\right) \in A\right] d x
\end{aligned}
$$

Lemma 4. Denoting by $v_{\mathcal{G}^{\prime}}$ the value of the game $\mathcal{G}^{\prime}$, we have

$$
v_{\mathcal{G}^{\prime}}-10 \kappa(b T)^{2} \ln \frac{1}{\kappa} \leq v_{\mathcal{G}} \leq v_{\mathcal{G}^{\prime}}+10 \kappa(b T)^{2} \ln \frac{1}{\kappa}
$$

and a strategy $\sigma$ guaranteeing reachability probability $v$ in $\mathcal{G}^{\prime}$, guarantees in $\mathcal{G}$ reachability probability in the interval $\left[v-10 \kappa(b T)^{2} \ln \frac{1}{\kappa}, v+10 \kappa(b T)^{2} \ln \frac{1}{\kappa}\right]$.

Proof. For a fixed continuous density function $f$, we first define the set of simulable points in $\mathcal{G}$ and $\mathcal{G}^{\prime}$ denoted $R_{f}, R_{f}^{\prime} \subseteq\left\{(r, x) \mid r \in \mathbb{R}_{\geq 0}, 0<x<f(r)\right\}$, respectively. A point $(r, x)$ is simulable in $\mathcal{G}$ if $r \geq \kappa$ and $x \leq f\left(r^{\prime}\right)$ for any $r^{\prime} \in[r-\kappa, r]$. A point $\left(r^{\prime}, x^{\prime}\right)$ is simulable in $\mathcal{G}^{\prime}$ if $x^{\prime} \leq f(r)$ for $r \in\left[r^{\prime}, r^{\prime}+\kappa\right]$.

We define a set of runs $X$ and $Y$ such that whenever up to time $T$ a timed transition in the specification is taken, the newly randomly generated pair $(r, x)$ is simulable in $\mathcal{G}$ and simulable in $\mathcal{G}^{\prime}$, respectively (by generating in $\mathcal{G}^{\prime}$ we mean the number that is randomly picked, not the shifted number that is actually stored in the state space). Next, we define a set of runs $Z$ such that at most one Markovian transition occurs in each interval $[\ell \kappa,(\ell+1) \kappa]$ for $0 \leq \ell<T / \kappa$.

Now we show that for any strategy $\sigma$ and $\pi$ it holds

$$
\begin{aligned}
\mathbf{P}_{\mathcal{G}^{\prime}}^{\sigma, \pi}\left[\diamond^{\leq T} G \cap X\right] & \leq \mathbf{P}_{\mathcal{G}}^{\sigma, \pi}\left[\nabla^{\leq T} G \mid Z\right] \\
\mathbf{P}_{\mathcal{G}^{\prime}}^{\sigma, \pi}\left[{ }^{\leq T} G\right] & \geq \mathbf{P}_{\mathcal{G}}^{\sigma, \pi}\left[\nabla^{\leq T} G \cap Y \mid Z\right]
\end{aligned}
$$


Conditioning by $Z$ only equalizes the behavior of the Markovian transitions as in $\mathcal{G}^{\prime}$ it is set by definition. The inequalities are obtained by the following idea: Whenever in $\mathcal{G}^{\prime}$ a number $z\left(=b+\kappa-r_{n}\right)$ is added to the randomly generated waiting time $r$, the behavior is the same is when in $\mathcal{G}$ the number $r+z$ is randomly generated. For each run in $X$ holds that the same run is also a run in $\mathcal{G}^{\prime}$ such that these (identical) runs are equivalent w.r.t. the time-bounded reachability. Furthermore, this mapping preserves measure due to the simulability of the choices in the specification. To each simulable choice $(r, x)$ in $\mathcal{G}$, and any shift $z \in[-\kappa, 0]$, there is enough marginal density to generate $(r-z, \cdot)$ in $\mathcal{G}^{\prime}$. The same arguments hold vice versa for $\mathcal{G}^{\prime}$.

From (3), we get

$$
\begin{aligned}
\mathbf{P}_{\mathcal{G}^{\prime}}^{\sigma, \pi}\left[\diamond^{\leq T} G \cap X\right] & \leq \mathbf{P}_{\mathcal{G}}^{\sigma, \pi}\left[\diamond^{\leq T} G \cap Z\right] / \mathbf{P}_{\mathcal{G}}^{\sigma, \pi}[Z] \\
\mathbf{P}_{\mathcal{G}^{\prime}}^{\sigma, \pi}\left[\diamond^{\leq T} G\right]-\mathbf{P}_{\mathcal{G}^{\prime}}^{\sigma, \pi}\left[\diamond^{\leq T} G \cap \neg X\right] & \leq \mathbf{P}_{\mathcal{G}}^{\sigma, \pi}\left[\diamond^{\leq T} G \cap Z\right] / \mathbf{P}_{\mathcal{G}}^{\sigma, \pi}[Z] \\
\mathbf{P}_{\mathcal{G}^{\prime}}^{\sigma, \pi}\left[{ }^{\leq T} G\right] & \leq \mathbf{P}_{\mathcal{G}}^{\sigma, \pi}\left[\diamond^{\leq T} G\right] / \mathbf{P}_{\mathcal{G}}^{\sigma, \pi}[Z]+\mathbf{P}_{\mathcal{G}^{\prime}}^{\sigma, \pi}[\neg X] \\
\mathbf{P}_{\mathcal{G}^{\prime}}^{\sigma, \pi}\left[{ }^{\leq T} G\right] & \leq \mathbf{P}_{\mathcal{G}}^{\sigma, \pi}\left[\diamond^{\leq T} G\right]+2\left(1-\mathbf{P}_{\mathcal{G}}^{\sigma, \pi}[Z]\right)+\left(1-\mathbf{P}_{\mathcal{G}^{\prime}}^{\sigma, \pi}[X]\right)
\end{aligned}
$$

where the last manipulation holds for $\mathbf{P}_{\mathcal{G}}^{\sigma, \pi}[Z]>1 / 2$. Similarly from (44), we get

$$
\begin{aligned}
\mathbf{P}_{\mathcal{G}}^{\sigma, \pi}\left[\diamond^{\leq T} G \cap Y \cap Z\right] / \mathbf{P}_{\mathcal{G}}^{\sigma, \pi}[Z] & \leq \mathbf{P}_{\mathcal{G}^{\prime}}^{\sigma, \pi}\left[{ }^{\leq T} G\right] \\
\mathbf{P}_{\mathcal{G}}^{\sigma, \pi}\left[\diamond^{\leq T} G\right] & \leq \mathbf{P}_{\mathcal{G}^{\prime}}^{\sigma, \pi}\left[{ }^{\leq T} G\right] \cdot \mathbf{P}_{\mathcal{G}}^{\sigma, \pi}[Z]+\mathbf{P}_{\mathcal{G}}^{\sigma, \pi}\left[{ }^{\leq T} G \cap(\neg Y \cup \neg Z)\right] \\
\mathbf{P}_{\mathcal{G}}^{\sigma, \pi}\left[\diamond^{\leq T} G\right] & \leq \mathbf{P}_{\mathcal{G}^{\prime}}^{\sigma, \pi}\left[{ }^{\leq T} G\right]+\mathbf{P}_{\mathcal{G}}^{\sigma, \pi}[\neg Y \cup \neg Z] \\
\mathbf{P}_{\mathcal{G}}^{\sigma, \pi}\left[\diamond^{\leq T} G\right] & \leq \mathbf{P}_{\mathcal{G}^{\prime}}^{\sigma, \pi}\left[\diamond^{\leq T} G\right]+\left(1-\mathbf{P}_{\mathcal{G}}^{\sigma, \pi}[Y]\right)+\left(1-\mathbf{P}_{\mathcal{G}}^{\sigma, \pi}[Z]\right)
\end{aligned}
$$

Finally, we need to bound $\left(1-\mathbf{P}_{\mathcal{G}}^{\sigma, \pi}[X]\right),\left(1-\mathbf{P}_{\mathcal{G}}^{\sigma, \pi}[Y]\right)$, and $\left(1-\mathbf{P}_{\mathcal{G}}^{\sigma, \pi}[Z]\right)$. As regards $\mathbf{P}_{\mathcal{G}}^{\sigma, \pi}[Z]$, notice that there are $T / \kappa$ intervals of length $\kappa$. Due to the memoryless property of the exponential distribution, we can bound the probability by summing $T / \kappa$ times the probability $p$ that in one interval there are two or more Markovian transitions.

$$
\left(1-\mathbf{P}_{\mathcal{G}}^{\sigma, \pi}[Z]\right) \leq \frac{T}{\kappa} \cdot p
$$

As the worst case we assume rate $b$ which bounds the maximal rate of $\mathcal{C}$. The probability $p$ can be bounded by

$$
\leq \frac{T}{\kappa} \cdot \frac{(b \kappa)^{2}}{2} \leq \frac{1}{2} \kappa b^{2} T
$$

which follows from the properties of the Poisson distribution with parameter $b \kappa$ using the very same arguments as in [M.R10, Lemma 6.2]. As regards $\mathbf{P}_{\mathcal{G}}^{\sigma, \pi}[X]$ and $\mathbf{P}_{\mathcal{G}}^{\sigma, \pi}[Y]$ we first bound how many times a timed transition in the specification can be taken. We assume the fastest possible transitions, i.e. uniformly 
distributed on $[0,1 / b]$ (recall that $b$ bounds the maximal density of transitions in $\mathcal{S}$ ). Let us express the probability $q$ that no more than $K=2 b T\left(1+\ln \frac{1}{\kappa}\right)$ transitions occur within time $T$, i.e. that the sum of the random times of the first $K$ transitions exceed $T$. As the expected value of this sum is $T\left(1+\ln \frac{1}{\kappa}\right)$, we can use the Hoeffding's inequality to bound the probability that the sum is not lower than its expected value by more than $T \ln \frac{1}{\kappa}$ )

$$
q \leq \exp \left(-\frac{2\left(T \ln \frac{1}{\kappa}\right)^{2}}{2 b T\left(1+\ln \frac{1}{\kappa}\right) \cdot(1 / b)^{2}}\right)=\exp \left(-\frac{T b \ln \frac{1}{\kappa}}{2}\right)=\kappa^{T b / 2} \leq \kappa
$$

due to the assumption that $b>2 / T$. Notice that the first manipulation holds for $\kappa \leq 1 / 3$ which we can easily assume. Further, observe that the probabilities $r, r^{\prime}$ that in one transition a point is sampled that is not simulable in $\mathcal{G}$ and $\mathcal{G}^{\prime}$, respectively, is bounded by $r \leq \kappa \cdot b+T \kappa b \leq 2 T \kappa b$ and $r^{\prime} \leq T \kappa b$ as $b$ bounds the maximal density as well as the maximal derivation of the densities in $\mathcal{S}$ (we further assume that $T \geq 1$. Hence,

$$
\begin{aligned}
\left(1-\mathbf{P}_{\mathcal{G}}^{\sigma, \pi}[X]\right) & \leq \kappa+K(2 T \kappa b)=\kappa+\left(2 b T\left(1+\ln \frac{1}{\kappa}\right)\right)(2 T \kappa b) \\
& \leq \kappa+8 \kappa(b T)^{2} \ln \frac{1}{\kappa} \leq 9 \kappa(b T)^{2} \ln \frac{1}{\kappa},
\end{aligned}
$$

and the same bound holds for $\left(1-\mathbf{P}_{\mathcal{G}}^{\sigma, \pi}[Y]\right)$, as well. All in all, from (5), (6) , (17), and (8) we obtain the lemma.

\section{E.2 The discrete-step game $\mathcal{G}^{\prime \prime}$}

The goal is to obtain a game very close to the discretized game $\Delta$. Conceptually, $\mathcal{G}^{\prime \prime}$ differs only a little from the game $\mathcal{G}^{\prime}$. There are two differences:

- every $\kappa$ time, there is a self-loop transition which materializes the grid introduced in $\mathcal{G}^{\prime}$, we call these self-loops artificial ticks;

- in a timed state at time $\ell \kappa+x$ for $\ell \in \mathbb{N}_{0}$ and $x \in[0, \kappa)$, the player env has two additional actions: 0 , and $\rightarrow(\kappa-x)$ which means playing almost $\kappa-x$, i.e. almost the time that remains until the grid line. The reason for these two actions is that optimizing the behavior in $\mathcal{G}^{\prime}$ may force the player to take an as small number as possible, or a number as close to the grid line from left as possible. The set of strategies in $\mathcal{G}^{\prime \prime}$, denoted by $\bar{\Pi}$, thus simplifies the notion of optimality to be transfered to the discrete game $\Delta$.

Histories in $\mathcal{G}^{\prime \prime}$ are $\mathbb{H}$ istories $\left(\mathcal{G}^{\prime \prime}\right)=\left(G \times\left(\mathbb{R}_{\geq 0} \cup\left\{\rightarrow(\ell \kappa) \mid \ell \in \mathbb{N}_{0}\right\}\right)\right)^{*} \times G$, where $\rightarrow x$ denotes almost time $x$. Algebraically, $\stackrel{\rightarrow}{\rightarrow}=x$, the only difference is that player con takes at time $\rightarrow(\ell \kappa)$ decision as in the interval $[(\ell-1) \kappa, \ell \kappa)$. To this end, $\lfloor\mathfrak{h}\rfloor$ has all $\rightarrow(\ell \kappa)$ replaced by $(\ell-1) \kappa$.

Let us fix a history $\mathfrak{h}=\left(v_{0}, r_{0}, u_{0}\right) t_{1} \cdots t_{n}\left(v_{n}, r_{n}, u_{n}\right)$ where each $v_{i} \in G_{1}$, $r_{i} \in \mathbb{R}_{\geq 0} \cup\left\{\rightarrow(\ell \kappa) \mid \ell \in \mathbb{N}_{0}\right\}$, and $u_{i} \in \mathbb{R}_{\geq 0}$. We fix a measurable set $A$ of histories. Further, let $a=0$ if $t_{n}=\rightarrow(\ell \kappa)$ for some $\ell \in \mathbb{N}$. Otherwise, let $a$ be the minimal number such that $t_{n}+a=\ell \kappa$ for some $\ell \in \mathbb{N}$, i.e. the remaining time till a grid line and $b$ be as in the definition of $\mathcal{G}^{\prime}$. 
- If $v_{n}$ is an immediate state, let $d=\sigma(\lfloor\mathfrak{h}\rfloor)$ and $e=\pi(\mathfrak{h})$. We have

$$
\begin{aligned}
P^{\prime \prime}(\mathfrak{h}, A)= & \sum_{v \in \operatorname{supp}(e)} e(v) \cdot\left[\mathfrak{h} t_{n}\left(v, r_{n}, u_{n}\right) \in A\right] \\
& +e(\checkmark) \cdot \sum_{v_{i} \stackrel{\operatorname{con}}{\hookrightarrow} v_{n+1}} d\left(v_{n+1}\right) \cdot\left[\mathfrak{h} t_{n}\left(v_{n+1}, r_{n}, u_{n}\right) \in A\right]
\end{aligned}
$$

- If $v_{n}$ is a timed state, let $F=\pi(\mathfrak{h})$. We distinguish four situations. For the first timed state visited after a new specification state is entered, we have

$$
P^{\prime \prime}(\mathfrak{h}, A)=\int_{0}^{\infty} \int_{0}^{\infty} u d\left(r^{\prime}, u^{\prime}\right) \cdot\left[\mathfrak{h}\left(t_{n}\right)\left(v_{n},\left(r^{\prime}+b+\kappa-r_{n}\right), u^{\prime}\right) \in A\right] d r^{\prime} d u^{\prime}
$$

where $u d$ is the density of the uniform distribution over the area $\{(r, x) \mid 0<$ $x<f(r)\}$ below the curve of $f$ where $f$ is the density of the distribution $d$. If a Markovian transition occured in $\mathfrak{h}$ since the last artificial tick, we have

$$
P^{\prime \prime}(\mathfrak{h}, A)=\int_{t_{e} \in \mathbb{R}^{\prime}} F\left(d t_{e}\right) \cdot\left(E^{\prime}\left(t_{e}\right) \cdot\left[t_{e}<a \vee t_{e}=\rightarrow a\right]+T^{\prime}(a) \cdot\left[t_{e} \geq a\right]\right)
$$

i.e. no Markovian transition can occur; similarly if $r_{n}<a$, we have

$$
P^{\prime \prime}(\mathfrak{h}, A)=\int_{t_{e} \in \mathbb{R}^{\prime}} F\left(d t_{e}\right) \cdot\left(E^{\prime}\left(t_{e}\right) \cdot\left[t_{e}<r_{n}\right]+S^{\prime}\left(r_{n}\right) \cdot\left[t_{e} \geq r_{n}\right]\right)
$$

i.e. no Markovian transition and no artificial tick can occur as first; otherwise

$$
\begin{gathered}
P^{\prime \prime}(\mathfrak{h}, A)=\int_{t_{e} \in \mathbb{R}^{\prime}} F\left(d t_{e}\right) \cdot\left(E\left(t_{e}\right) \cdot\left[t_{e}<a \vee t_{e}=\rightarrow a\right]+T(a) \cdot\left[t_{e} \geq a\right]\right. \\
\left.+\sum_{\substack{\lambda \\
v_{i} \rightsquigarrow v}} M_{v}\left(\min \left\{t_{e}, a\right\}\right)\right)
\end{gathered}
$$

where $\mathbb{R}^{\prime}=\mathbb{R}_{\geq 0} \cup\{\rightarrow a\}$, the term $T(a)$ describes the impact of the the artificial tick, the terms $E^{\prime}, S^{\prime}$, and $T^{\prime}$ describe the situation where there is no Markovian transition to compete with

$$
\begin{aligned}
T^{\prime}(a) & =\left[\mathfrak{h}\left(t_{n}+a\right)\left(v_{n}, r_{n}-a, u_{n}\right) \in A\right], \\
E^{\prime}(t) & =\left[\mathfrak{h}\left(t_{n}+t\right)\left(v^{\prime}, r_{n}-t, u_{n}\right) \in A\right], \\
S^{\prime}(t) & =\left[\mathfrak{h}\left(t_{n}+t\right)\left(v^{\prime \prime}, 0, u_{n}\right) \in A\right],
\end{aligned}
$$

and $T(a)=e^{-\mu a} \cdot T^{\prime}(a)$ where $\mu=\sum_{v_{n} \rightsquigarrow v_{n+1}} \lambda, v_{n} \stackrel{\text { Change }}{\hookrightarrow} v^{\prime}$, and $v_{n} \stackrel{d}{\rightsquigarrow} v^{\prime \prime}$. 
Lemma 5. The game $\mathcal{G}^{\prime}$ has the same value as the game $\mathcal{G}^{\prime \prime}$, i.e.

$$
\sup _{\sigma \in \Sigma} \inf _{\pi \in \Pi} \mathcal{P}_{\mathcal{G}^{\prime}}^{\sigma, \pi}\left[\diamond^{\leq T} G\right]=\sup _{\sigma \in \Sigma} \inf _{\pi \in \bar{\Pi}} \mathcal{P}_{\mathcal{G}^{\prime \prime}}^{\sigma, \pi}\left[\diamond^{\leq T} G\right]
$$

and a strategy $\sigma$ guarantees both in $\mathcal{G}^{\prime}$ and $\mathcal{G}^{\prime \prime}$ the same value.

Proof. We fix a strategy $\sigma \in \Sigma$. As regards $\geq$, the only change are the artificial ticks. The strategy $\pi$ of env simulates in history $\mathfrak{h}$ in $\mathcal{G}^{\prime \prime}$ what the strategy $\pi^{\prime}$ of env does in history $\mathfrak{h}^{\prime}$ in $\mathcal{G}^{\prime}$, where $\mathfrak{h}^{\prime}$ is obtained from $\mathfrak{h}$ by removing the artificial ticks. In immediate states, simply $\pi(\mathfrak{h})=\pi\left(\mathfrak{h}^{\prime}\right)$. In timed states the distribution on time $\pi(\mathfrak{h})$ is obtained from $\pi\left(\mathfrak{h}^{\prime}\right)$ by conditioning by the amount of time $\left(t-t^{\prime}\right)$ that has been already spent waiting where $t$ and $t^{\prime}$ is the total time of $\mathfrak{h}$ and $\mathfrak{h}^{\prime}$, respectively. This way, we get an obvious correspondence of runs that preserves measure, i.e. we obtain completely the same probability to reach the target in $\mathcal{G}^{\prime \prime}$ as in $\mathcal{G}^{\prime}$.

Ar regards $\leq$, for a strategy $\pi$ in $\mathcal{G}^{\prime \prime}$ a sequence of strategies $\left(\pi_{i}\right)_{i \in \mathbb{N}}$ is defined that wait $\delta /\left(i \cdot 2^{j}\right)$ instead of $0^{\leftarrow}$ and $x-\delta /\left(i \cdot 2^{j}\right)$ instead of $\rightarrow x$ in the $j$-th step. After such an imprecise waiting, the strategy further simulates what $\pi$ would do if it waits precisely $0^{\leftarrow}$ or $\rightarrow x$. I.e. for each history $\mathfrak{h}$ in $\mathcal{G}^{\prime}$, the simulating strategy uses the decisions of $\pi\left(\mathfrak{h}^{\prime}\right)$ where $\mathfrak{h}^{\prime}$ is obtained from $\mathfrak{h}$ by inserting the artificial ticks and replacing the imprecise waiting by the precise waiting as chosen by $\pi$. Furthermore, observe that the waiting of $\pi$ gets interrupted by the artificial ticks, i.e. its plans beyond the closes tick are irrelevant. Each $\pi_{i}$ has to plan the waiting in advance, i.e. connects the waiting distributions of $\pi$ in the current moment, after one artificial tick, after two artificial ticks, etc., as follows. Let $(v, r)$ be the last state of $\mathfrak{h}^{\prime}$ and let $t$ and $t^{\prime}$ denote the total time of $\mathfrak{h}$ and $\mathfrak{h}^{\prime}$ and $x, x^{\prime} \in \mathbb{R}_{>0}$ be the minimal numbers such that $t+x=\delta k$ and $t^{\prime}+x^{\prime}=\delta k^{\prime}$ for some $k . k^{\prime} \in \mathbb{N}$. The distribution $\pi_{i}(\mathfrak{h})$ is defined

- on $\left[\left(t^{\prime}-t\right),\left(t^{\prime}-t\right)+x^{\prime}\right]$ using $\pi\left(\mathfrak{h}^{\prime}\right)$ on $\left[0, x^{\prime}\right]$ (if $\left(t^{\prime}-t\right)$ is negative, the distribution set to negative numbers is concentrated on $\delta /\left(i \cdot 2^{j}\right)$ instead),

- on $\left[\left(t^{\prime}-t\right)+x^{\prime},\left(t^{\prime}-t\right)+x^{\prime}+\delta\right]$ using $\pi\left(\mathfrak{h}^{\prime}\left(t+x^{\prime}\right)\left(v, r-t-x^{\prime}\right)\right)$ on $[0, \delta]$ conditioned by the waiting step not being taken in the interval $\left[0, x^{\prime}\right]$,

- on $\left[\left(t^{\prime}-t\right)+x^{\prime}+\delta,\left(t^{\prime}-t\right)+x^{\prime}+2 \delta\right]$ using $\pi\left(\mathfrak{h}^{\prime}\left(t+x^{\prime}\right)\left(v, r-t+x^{\prime}\right)(t+\right.$ $\left.\left.x^{\prime}+\delta\right)\left(v, r-t-x^{\prime}-\delta\right)\right)$ on $[0, \delta]$ conditioned by the waiting step not being taken in the interval $\left[0, x^{\prime}+\delta\right]$, etc.

The behavior of $\mathcal{G}^{\prime}$ and $\mathcal{G}^{\prime \prime}$ differs only if a Markovian transition occurs within the imprecision in waiting. Since the total sum of the imprecision in waiting on any run is at most $\delta / i$, the measure of runs that differs tends to 0 as $i \rightarrow \infty$.

\section{E.3 Grid strategies in $\mathcal{G}^{\prime \prime}$}

We say that two histories $\mathfrak{h}$ and $\mathfrak{h}^{\prime}$ follow the same pattern, denoted $\mathfrak{h} \sim \mathfrak{h}^{\prime}$ if $\mathfrak{h}=\left(v_{0}, r_{0}, u_{0}\right) t_{1} \ldots t_{n}\left(v_{n}, r_{n}, u_{n}\right), \mathfrak{h}^{\prime}=\left(v_{0}^{\prime}, r_{0}^{\prime}, u_{0}^{\prime}\right) t_{1}^{\prime} \ldots t_{n}^{\prime}\left(v_{n}^{\prime}, r_{n}^{\prime}, u_{n}^{\prime}\right)$ with $v_{i}=$ $v_{i}^{\prime}$ and $t_{i}$ and $t_{i}^{\prime}$ as well as $r_{i}$ and $r_{i}^{\prime}$ equal when rounded down to a multiple of $\kappa$ for all $1 \leq i \leq n$.

We say that a strategy $\pi$ of $\mathcal{E}$ is a grid strategy, denoted $\pi \in \bar{\Pi}_{\delta}$, if 
1. the strategy is deterministic;

2. for any history $\mathfrak{h}$ of time $t$ ending in a timed state, $t+\pi(\mathfrak{h})=k \cdot \delta$ for $k \in \mathbb{N}$;

3 . for any histories $\mathfrak{h}$ of time $t$ and $\mathfrak{h}^{\prime}$ of time $t^{\prime}$ that follow the same pattern, we have $\pi(\mathfrak{h})=\pi\left(\mathfrak{h}^{\prime}\right)$ if $\mathfrak{h}$ ends in an immediate state, and $t+\pi(\mathfrak{h})=t^{\prime}+\pi\left(\mathfrak{h}^{\prime}\right)$ if $\mathfrak{h}$ ends in a timed state ( $\pi$ plans the Change at the same absolute time).

Lemma 6. In the game $\mathcal{G}^{\prime \prime}$, grid strategies suffice for player env, i.e.

$$
\sup _{\sigma \in \Sigma} \inf _{\pi \in \bar{\Pi}} \mathcal{P}_{\mathcal{G}^{\prime \prime}}^{\sigma, \pi}\left[\diamond^{\leq T} G\right]=\sup _{\sigma \in \Sigma} \inf _{\pi \in \bar{\Pi}_{\delta}} \mathcal{P}_{\mathcal{G}^{\prime \prime}}^{\sigma, \pi}\left[\diamond^{\leq T} G\right]
$$

Proof. First, observe that for each $\varepsilon>0$ there is $n \in \mathbb{N}$ such that

$$
\sup _{\sigma \in \Sigma} \inf _{\pi \in \bar{\Pi}} \mathcal{P}_{\mathcal{G}^{\prime \prime}}^{\sigma, \pi}\left[\diamond_{\leq n}^{\leq T} G\right] \leq \sup _{\sigma \in \Sigma} \inf _{\pi \in \bar{\Pi}} \mathcal{P}_{\mathcal{G}^{\prime \prime}}^{\sigma, \pi}\left[\diamond^{\leq T} G\right]+\varepsilon
$$

where $\diamond \leq \sum_{n} G$ is the set of runs that reach the target within $n$ discrete steps within time $T$. Indeed, the probability of $n$ Markovian transitions to occur within time $T$ tends to 0 as $n \rightarrow \infty$, the number of internal transitions in $\mathcal{C}$ that can be performed between two Markovian transitions is limited by the acyclicity assumption, and the set of runs where $\pi$ makes infinitely many transitions within time $T$ is 0 .

Thanks to this fact, it is enough to show that for any $n$ the grid strategies suffice for the $n$-step time bounded reachability. Let us fix $\sigma$ and $n \in \mathbb{N}$. We show that there is a grid strategy $\pi^{*}$ which is optimal w.r.t. $n$-steps, i.e.

$$
\mathcal{P}_{\mathcal{G}^{\prime \prime}}^{\sigma, \pi^{*}}\left[\diamond_{\leq n}^{\leq T} G\right]=\inf _{\pi \in \bar{\Pi}} \mathcal{P}_{\mathcal{G}^{\prime \prime}}^{\sigma, \pi}\left[\diamond_{\leq n}^{\leq T} G\right]
$$

We construct $\pi^{*}$ by induction on the number of steps already performed in a history $\mathfrak{h}$, denoted $|\mathfrak{h}|$. Along the construction, we also prove the following claim

Claim. Let $0 \leq i \leq n$ and $\mathfrak{h}$ be an $i$-step history.

1. If $\mathfrak{h}$ has total time $k \delta$ for some $k \in \mathbb{N}$,

$$
\mathcal{P}_{\mathcal{G}^{\prime \prime}, \mathfrak{h}}^{\sigma, \pi^{*}}\left[\diamond_{\leq n}^{\leq T} G\right]=\inf _{\pi \in \bar{\Pi}} \mathcal{P}_{\mathcal{G}^{\prime \prime}, \mathfrak{h}}^{\sigma, \pi}\left[\diamond_{\leq n}^{\leq T} G\right]
$$

2. If a Markovian transition already occurred in $\mathfrak{h}$ since the last artificial tick,

$$
\mathcal{P}_{\mathcal{G}^{\prime \prime}, \mathfrak{h}}^{\sigma, \pi^{*}}\left[\diamond_{\leq n}^{\leq T} G\right]=\inf _{\pi \in \bar{\Pi}} \inf _{\mathfrak{h}^{\prime} \sim \mathfrak{h}} \mathcal{P}_{\mathcal{G}^{\prime \prime}, \mathfrak{h}^{\prime}}^{\sigma, \pi}\left[\diamond_{\leq n}^{\leq T} G\right]
$$

Here, $\mathcal{P}_{\mathcal{G}^{\prime \prime}, \mathfrak{h}}^{\sigma, \pi}$ is the probability measure of the chain where $\mathfrak{h}$ is the initial state. Note that the second point of the claim applies to two situations: in the current "grid slot", either a Markovian transition already occurred, or the specification state is about to change.

As the induction base, we take arbitrary grid strategy $\pi^{*}$. For $i=n$, the claim obviously holds. For the induction step, we assume that $\pi^{*}$ satisfies the claim for all $i>j$. We alter $\pi^{*}$ to be satisfy the claim for $i=j$ as well. First, let $\mathfrak{h}$ be a $i$-step history of total time $k \delta$ for some $k \in \mathbb{N}$. Let $\mathfrak{h}$ end in a state $(v, r)$. 
- If $v$ is immediate, we set $\pi(\mathfrak{h})$ to the action that minimizes

$$
\min \left\{\sum_{v \stackrel{\text { con }}{\hookrightarrow} v^{\prime}} \sigma(\mathfrak{h})\left(v^{\prime}\right) \cdot \mathcal{P}_{\mathcal{G}^{\prime \prime}, \mathfrak{h} t\left(v^{\prime}, r\right)}^{\sigma, \pi^{*}}\left[\diamond_{\leq n}^{\leq T} G\right], \min _{v \stackrel{\text { env }}{\hookrightarrow} v^{\prime}} \mathcal{P}_{\mathcal{G}^{\prime \prime}, \mathfrak{h} t\left(v^{\prime}, r\right)}^{\sigma, \pi^{*}}\left[\diamond_{\leq n}^{\leq T} G\right]\right\},
$$

where the first choice corresponds to the action $\checkmark$.

- If $v$ is timed and a Markovian transition can occur before the next artificial tick, we set $\pi(\mathfrak{h})$ to $0^{\leftarrow}, \rightarrow \delta$, or $\delta$ depending on which minimizes

$\min \left\{\mathcal{P}_{\mathcal{G}^{\prime \prime}, \mathfrak{h} t\left(v^{\prime \prime}, r\right)}^{\sigma, \pi^{*}}\left[\diamond_{\leq n}^{\leq T} G\right], \mathcal{A}+\mathcal{P}_{\mathcal{G}^{\prime \prime}, \mathfrak{h} \bar{t}\left(v^{\prime \prime}, r-\delta\right)}^{\sigma, \pi^{*}}\left[\diamond_{\leq n}^{\leq T} G\right], \mathcal{A}+\mathcal{P}_{\mathcal{G}^{\prime \prime}, \mathfrak{h} t(v, r-\delta)}^{\sigma, \pi^{*}}\left[\diamond_{\leq n}^{\leq T} G\right]\right\}$

where $v \stackrel{\text { Change }}{\hookrightarrow} v^{\prime \prime}$ and $\mathcal{A}=\int_{0}^{\delta} \mu \cdot e^{-\mu x} \sum_{v \leadsto v^{\prime}} \frac{\lambda}{\mu} \cdot \mathcal{P}_{\mathcal{G}^{\prime \prime}, \mathfrak{h} t+x\left(v^{\prime}, r-x\right)}^{\sigma, \pi^{*}}\left[\diamond_{\leq n}^{\leq T} G\right] d x$.

- If $v$ is timed and the specification state is about to change before the next artificial tick, i.e. at time $b<\delta$, we set $\pi(\mathfrak{h})$ to $0^{\leftarrow}$ or to $\delta$, depending on which minimizes

$$
\min \left\{\mathcal{P}_{\mathcal{G}^{\prime \prime}, \mathfrak{h} t\left(v^{\prime \prime}, r\right)}^{\sigma, \pi^{*}}\left[\diamond_{\leq n}^{\leq T} G\right], \int_{0}^{\infty} f(x) \cdot \mathcal{P}_{\mathcal{G}^{\prime \prime}, \mathfrak{h} t+b\left(v^{\prime \prime \prime}, x\right)}^{\sigma, \pi^{*}}\left[\vartheta_{\leq n}^{\leq T} G\right] d x,\right\}
$$

where $v^{\prime \prime \prime}$ is reached from $v$ when the specification changes state and $f$ is the density of waiting in the new specification state.

Second, let $\mathfrak{h}$ be a $i$-step history of total time $t$ where a Markovian transition already occurred since the last artificial tick. Let $\mathfrak{h}$ end in a state $(v, r)$ and let $a$ be minimal such that $t+a=k \delta$ for some $k \in \mathbb{N}$.

- If $v$ is immediate, we again set $\pi(\mathfrak{h})$ to the action that minimizes

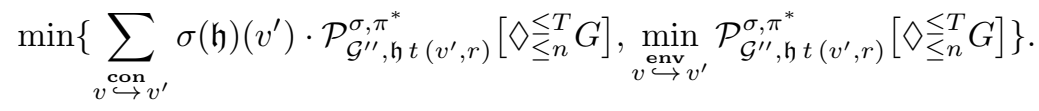

- If $v$ is timed, we set $\pi(\mathfrak{h})$ to $\rightarrow a$ or to $a$ depending on which minimizes

$$
\min \left\{\mathcal{P}_{\mathcal{G}^{\prime \prime}, \mathfrak{h} t+\bar{a}\left(v^{\prime \prime}, r-a\right)}^{\sigma, \pi^{*}}\left[\diamond_{\leq n}^{\leq T} G\right], \mathcal{P}_{\mathcal{G}^{\prime \prime}, \mathfrak{h} t+a(v, r-a)}^{\sigma, \pi^{*}}\left[\diamond_{\leq n}^{\leq T} G\right]\right\}
$$

For all remaining $i$-step histories we set the strategy so that it is a grid strategy.

As regards the second point of the claim, observe that all the reachability probabilities are from longer histories, i.e. they do not depend on exact timing of $\mathfrak{h}$. Hence, also the choice and the reachability probability in the $i$-th step does not depend on the exact timing, it cannot be lower for any $\mathfrak{h}^{\prime} \sim \mathfrak{h}$. Observe that for an immediate state, no other choice (possibly mixing among the pure choices) can yield a lower reachability probability. For a timed state, the chain must move either to history $\mathfrak{h} t+a(v, r-a)$ (no action taken until the artificial tick) or to a history of the form $\mathfrak{h} t+\bar{x}\left(v^{\prime \prime}, r-x\right)$ for some $x \leq a$ (Change taken at time $x$ ). From the induction hypothesis, the reachability probability does not depend on $x$, i.e. restricting to the pure choice $\rightarrow a$ does not hamper optimality and results in a grid strategy.

As regards the first point of the claim, we again fix an $i$-step history $\mathfrak{h}$ of total time $k \delta$ for some $k \in \mathbb{N}$. Let $\mathfrak{h}$ end in a state $(v, r)$. 
- If $v$ is immediate, again, no other choice (possibly mixing among the pure choices) can yield a lower reachability probability.

- If $v$ is timed and a Markovian transition can occur before the next artificial tick, the situation is much more complicated. First observe that when a Markovian transition occurs in state $v^{\prime}$ at time $x$, the (optimal) probability to reach the target does not depend on $x$ due to the second point of the claim. Thus, we can denote it $p_{v^{\prime}}$. Waiting in the interval $[a, b]$ in state $v^{\prime}$ for a Markovian transition to occur contributes to the reachability probability with $\int_{a}^{b} \mu \cdot e^{-\mu x} \cdot p_{v^{\prime}} d x=p_{v^{\prime}}\left(e^{-\mu a}-e^{-\mu b}\right)$. Hence optimizing the decisions in $v$ boils down to spending the $\delta$ time in such a state $v^{*}$ where the contribution is maximal, i.e. where $p_{v^{*}}$ is maximal; then before or after the next artificial tick moving to the state where the contribution is maximal for the following interval of size $\delta$ (the strategy $\sigma$ may take different actions before and after the artificial tick, hence we need to consider both options). Indeed, there is no reason to hesitate with moving to such $v^{*}$ as to probability to move to such a state also does not depend on time $x$ when the move is taken. Precisely, taking Change in state $v^{\prime}$ at time $x$ results in traversing a finite sequence of states in 0 time, ending in some timed state where a Markovian transition is again awaited. Importantly, the (possibly random) decisions of con in this sequence do not depend on $x$. All in all, action $0^{\leftarrow}$ is taken to change the current state, and actions $\rightarrow \delta$ or $\delta$ are taken if the contribution of the current state is optimal and the state is to be changed before or after the next artificial tick.

This concludes the proof of the claim as well as the proof of the lemma.

\section{E.4 Discrete game $\Delta$}

We will define the discrete game $\Delta$ as an extensive-form game KMvS94. As the game $\mathcal{G}^{\prime \prime}$ with a grid strategy has an almost discrete structure not much work is left to define the discrete game. Observe that for a grid strategy $\pi$ the behaviour of the Markov chain of $\mathcal{G}^{\prime \prime}$ in a history $\mathfrak{h}$ does not depend on exact timing of $\mathfrak{h}$, i.e. for all $\mathfrak{h}^{\prime} \sim \mathfrak{h}$ it holds $\mathcal{P}_{\mathcal{G}^{\prime \prime}, \mathfrak{h}}^{\sigma, \pi}\left[\oslash^{\leq T} G\right]=\mathcal{P}_{\mathcal{G}^{\prime \prime}, \mathfrak{h}^{\prime}}^{\sigma, \pi}[\diamond \leq T G]$. Hence, we can define the same game on the partition $V=\left\{[\mathfrak{h}]_{\sim} \mid \mathfrak{h} \in X\right\}$ of the set of histories $X \subset \mathbb{H}$ istories $\left(\mathcal{G}^{\prime \prime}\right)$ where the total time is $\leq T$ and that, intuitively speaking, can possibly be ever played. Formally $X$ are the histories where

- the total time is $\leq T$,

- there is at most one Markovian transition in each grid interval,

- the Change transitions are taken only at times $\ell \kappa$ or $\rightarrow(\ell \kappa)$.

Due to these restrictions, only a limited number of steps can be played up to time $T$, hence, $V$ is finite. For a vertex $v \in V$, we denote by $(\downarrow v)$ the last state of all the histories in the class $v$. To comply with the definition of extensive-form game, we divide the vertices where con takes decisions from the vertices where env takes decisions and from the stochastic vertices. Hence, we set

$$
V^{\prime}=V \cup V_{i} \times\{\checkmark\} \cup\left(V_{\vdash} \times\{0, \rightarrow \kappa, \kappa\} \cup V_{+} \times\{\rightarrow \kappa, \kappa\} \cup V_{\dashv}\right),
$$


where $V_{i}$ are the vertices corresponding to immediate states, $V_{\vdash}, V_{+}$, and $V_{\dashv}$ are the vertices corresponding to timed states with total time $\ell \kappa, \ell \kappa+x$ and $\rightarrow \ell \kappa$, respectively, for some $\ell \in \mathbb{N}_{0}$ and $x \in(0, \kappa)$. The vertices are divided among the players as follows.

$-V_{i} \cup V_{\vdash} \cup V_{+}$are the vertices of the first player (player env) with actions $\left\{v^{\prime} \in V \mid \operatorname{last}(v) \stackrel{\text { env }}{\hookrightarrow} \operatorname{last}\left(v^{\prime}\right)\right\} \cup\{\checkmark\}$ if $v \in V_{i},\{0, \kappa, \rightarrow \kappa\}$ if $v \in V_{\vdash}$, and $\{\kappa, \rightarrow \kappa\}$ if $v \in V_{+}$;

$-V_{i} \times\{\checkmark\}$ are the vertices of the second player (player con) with actions $\left\{v^{\prime} \in V \mid(\downarrow v) \stackrel{\text { con }}{\hookrightarrow}\left(\downarrow v^{\prime}\right)\right\}$ for any vertex $(v, \checkmark)$ of con;

$-V_{\vdash} \times\{0, \rightarrow \kappa, \kappa\} \cup V_{+} \times\{\rightarrow \kappa, \kappa\} \cup V_{\dashv}$ are the stochastic vertices.

Timed vertices with total time $T$ are the terminal vertices, denoted $Z$. For all other remaining vertices, the tree-like transition structure is defined as follows. In a vertex $v \in V$, any action $a \in\{\checkmark, 0, \rightarrow \kappa, \kappa\}$ leads to the vertex $(v, a)$. In a vertex $v \in V^{\prime}$, action $v^{\prime} \in V$ leads to the vertex $v^{\prime}$. The probability matrix $P^{\Delta}$ for the stochastic vertices is defined as follows.

- For $v \in V_{\dashv}$ with $\mathfrak{h} \in v$ and $v^{\prime} \in V^{\prime}$, we set $P^{\delta}\left(v, v^{\prime}\right)=P^{\prime \prime}\left(\mathfrak{h}, v^{\prime}\right)$ where $P^{\prime \prime}$ is induced by arbitrary strategies $\sigma$ and $\pi$.

- For $(v, a) \in\left(V_{\vdash} \times\{0, \rightarrow \kappa, \kappa\}\right) \cup\left(V_{+} \times\{\rightarrow \kappa, \kappa\}\right)$ with $\mathfrak{h} \in v$ and $v^{\prime} \in V^{\prime}$, we set $P^{\delta}\left((v, a), v^{\prime}\right)=P^{\prime \prime}\left(\mathfrak{h}, v^{\prime}\right)$ where $P^{\prime \prime}$ is induced by any strategy $\sigma$ and a grid strategy that chooses in $\mathfrak{h}$ action $a$.

Each terminal vertex $v \in Z$ has payoff $u(v)$ associated: vertex of histories that visit $G$ in the first component have payoff 1 , other vertices have payoff 0 . Recall that $\kappa=n \cdot \delta$. The observation sets for the first player are

$H_{1}=\left\{\left\{v \in V^{\prime} \mid(\downarrow v)=(c, s, e), t\right.\right.$ is the total time of $\left.\left.v,\lfloor t / \delta\rfloor=i\right\} \mid i \in \mathbb{N}_{0}, c \in C\right\}$,

i.e. the vertices in one observation set agree on the first component of the last state and on the total time up to the precision of $\delta$. Observation sets for the second player are singletons, i.e.

$$
H_{2}=\left\{\{v\} \mid v \in V^{\prime}\right\}
$$

A behavioural strategy of the first and the second player is a function that assigns to each observation of the player a probability distribution over actions available in this vertex. We denote these sets by $\Sigma_{\Delta}$ and $\Pi_{\Delta}$. Observe that the strategies of player con and grid strategies of player env in the game $\mathcal{G}^{\prime \prime}$ coincide with the strategies of the first and the second player in $\Delta$. The value of the game is defined as the expected payoff:

$$
\sup _{\sigma \in \Sigma_{\Delta}} \inf _{\pi \in \Pi_{\Delta}} E^{\sigma, \pi}[u] .
$$

Notice that this definition is equivalent to the definition via reachability in the main body. From these observations and from the construction we immediately get that the values equal:

Lemma 7. We have that $v_{\mathcal{G}^{\prime \prime}}=v_{\Delta}$ and any strategy of player 1 in $v_{\Delta}$ corresponds to a strategy of player con in $\mathcal{G}^{\prime \prime}$ guaranteeing the same value. 


\section{E.5 Solution of the discrete game $\Delta$}

Lemma 8. The value $v_{\Delta}$ and an optimal strategy can be computed in time polynomial in $|\Delta|$.

Proof. Because the observation sets of player 1 form a tree such that each action of player 1 results in a move in this tree, it is easy to see that $\Delta$ satisfies the condition of perfect recall (see KMvS94]). The value and the optimal strategies can be then computed using a linear program KMvS94 of size linear in the state space. 